\title{
Exponential asymptotics for intersection local times of stable processes and random walks
}

\author{
Xia Chen ${ }^{\mathrm{a}, 1}$, Jay Rosen ${ }^{\mathrm{b}, * 2}$ \\ a Department of Mathematics, University of Tennessee, Knoxville, TN 37996-1300, USA \\ ${ }^{\mathrm{b}}$ Department of Mathematics, College of Staten Island, CUNY, Staten Island, NY 10314, USA \\ Received 1 December 2003; received in revised form 4 August 2004; accepted 20 September 2004 \\ Available online 5 February 2005
}

\begin{abstract}
We study large deviations for intersection local times of $p$ independent $d$-dimensional symmetric stable processes of index $\beta$, under the condition $p(d-\beta)<d$. Our approach is based on Feynman-Kac type large deviations, moment computations and some techniques from probability in Banach spaces.
\end{abstract}

(C) 2005 Elsevier SAS. All rights reserved.

\section{Résumé}

On étudie les temps locaux d'intersection de $p$ processus $\beta$-stables $d$-dimensionnels indépendants, sous l'hypothèse $p(d-\beta)<d$. Notre approche est fondée sur les grandes déviations type Feynman Kac, des calculs de moments et quelques techniques de probabilités dans les espaces de Banach.

(c) 2005 Elsevier SAS. All rights reserved.

\section{Introduction}

Let $X(t)$ be a non-degenerate $d$-dimensional stable processes of index $\beta$. We assume that $X(t)$ is symmetric, i.e. $X(t) \stackrel{d}{=}-X(t)$, but we do not assume it is spherically symmetric. Thus

$$
E\left(\mathrm{e}^{\mathrm{i} \lambda \cdot X(t)}\right)=\mathrm{e}^{-t \psi(\lambda)}
$$

\footnotetext{
* Corresponding author.

E-mail addresses: xchen@math.utk.edu (X. Chen), jrosen3@earthlink.net (J. Rosen).

1 Research partially supported by NSF grant \#DMS-0405188.

2 Research partially supported by grants from the NSF and from PSC-CUNY. 
where $\psi(\lambda) \geqslant 0$ is continuous, positively homogeneous of degree $\beta$, i.e. $\psi(r \lambda)=r^{\beta} \psi(\lambda)$ for each $r \geqslant 0, \psi(-\lambda)=$ $\psi(\lambda)$, and for some $0<c<C<\infty$

$$
c|\lambda|^{\beta} \leqslant \psi(\lambda) \leqslant C|\lambda|^{\beta} .
$$

Let $X_{1}(t), \ldots, X_{p}(t)$ be independent copies of $X(t)$. Their ranges will have a point in common aside from the initial point if and only if $p(d-\beta)<d$, see [10]. When $p(d-\beta)<d$ there is a random measure $\alpha_{p}\left(\mathrm{~d} s_{1}, \ldots, \mathrm{d} s_{p}\right)$ supported on

$$
\left\{\left(t, \ldots, t_{p}\right) \in\left(\mathbb{R}^{+}\right)^{p} ; X_{1}\left(t_{1}\right)=\cdots=X_{p}\left(t_{p}\right)\right\} .
$$

$\alpha_{p}\left(\mathrm{~d} s_{1}, \ldots, \mathrm{d} s_{p}\right)$ is called the intersection local time of $X_{1}\left(t_{1}\right), \ldots, X_{p}\left(t_{p}\right)$. Formally it can be written as

$$
\alpha_{p}\left(\mathrm{~d} s_{1}, \ldots, \mathrm{d} s_{p}\right)=\left[\int_{\mathbb{R}^{d}} \prod_{j=1}^{p} \delta_{0}\left(X_{j}\left(s_{j}\right)-x\right) \mathrm{d} x\right] \mathrm{d} s_{1} \cdots \mathrm{d} s_{p}
$$

where $\delta_{0}(x)$ is the Dirac delta- 'function' at 0 .

In the case $d=1$ and $\beta>1$, the intersection local time can be represented in terms of the spatial $L^{p}\left(\mathbb{R}^{1}\right)$ norms of the local times $L_{t}^{x}$ of the symmetric stable processes. In this case the large deviations and law of the iterated logarithm have been established for a $\alpha_{p}(\cdot)$ in recent work [4] for Brownian motion and [5] for the symmetric stable processes.

When $d=1$ and $\beta \leqslant 1$, or when $d>1$ for all $\beta$, local time do not exist and we define the intersection local time $\alpha_{p}\left(\mathrm{~d} s_{1}, \ldots, \mathrm{d} s_{p}\right)$ as a limit. Let $h$ be a positive symmetric function in the Schwartz space $\mathcal{S}\left(\mathbb{R}^{d}\right)$ with $\int h \mathrm{~d} x=1$. Given $\epsilon>0$, let $h_{\epsilon}(x)=\epsilon^{-d} h(x / \epsilon)$, and define the random measure $\alpha_{p, \epsilon}(\cdot)$ on $\left(\mathbb{R}^{p}\right)^{+}$by

$$
\alpha_{p, \epsilon}\left(\mathrm{d} s_{1}, \ldots, \mathrm{d} s_{p}\right)=\left[\int_{\mathbb{R}^{d}} \prod_{j=1}^{p} h_{\epsilon}\left(X_{j}\left(s_{j}\right)-x\right) \mathrm{d} x\right] \mathrm{d} s_{1} \cdots \mathrm{d} s_{p} .
$$

It can be shown that if $p(d-\beta)<d$ the limit

$$
\alpha_{p}(B)=\lim _{\epsilon \rightarrow 0^{+}} \alpha_{p, \epsilon}(B)
$$

exists a.s. and in all $L^{m}$-norms for any $m \geqslant 1$ and any Borel set $B \subset\left(\mathbb{R}^{p}\right)^{+}$, and $\alpha_{p}(\cdot)$ is a measure supported on (1.3). We set $\alpha_{p, t}=\alpha_{p}\left([0, t]^{p}\right), \alpha_{p, t, \epsilon}=\alpha_{p, \epsilon}\left([0, t]^{p}\right)$. For the convenience of the reader we show in Theorem 9 that a.s. $\alpha_{p, t}=\lim _{\epsilon \rightarrow 0^{+}} \alpha_{p, t, \epsilon}$ exists and is continuous in $t$. Using the scaling property $\{X(t s) ; s \geqslant 0\} \stackrel{d}{=}$ $t^{1 / \beta}\{X(s) ; s \geqslant 0\}$ of the stable process it is easy to check that

$$
\alpha_{p, t}=t^{p-(p-1) d / \beta} \alpha_{p, 1}
$$

We note that in the case $\beta>d$, where local times exist, we can also consider the analogue of (1.4) where we use a single process rather than $p$ independent processes. Once again this is dealt with in [5]. However, in the case $\beta \leqslant d$ considered in this paper, where local times do not exist, if in (1.5) we use a single process rather than $p$ independent processes, the limit blows up. To get a non-trivial limit we must 'renormalize'. Large deviations for the resulting limit in the case $p=2$ are discussed in $[1,2]$.

To describe our results we need some further notation. For any function $f \in L^{2}\left(\mathbb{R}^{d}\right)$ set

$$
\mathcal{E}_{\psi}(f, f)=:(2 \pi)^{-d / 2} \int_{\mathbb{R}^{d}} \psi(\lambda)|\hat{f}(\lambda)|^{2} \mathrm{~d} \lambda
$$

where $\hat{f}(\lambda)$ denotes the Fourier transform of $f . \mathcal{E}_{\psi}(f, f)$ is the Dirichlet form of $\{X(t) ; t \geqslant 0\}$. Let

$$
\mathcal{F}_{\psi}=\left\{f \in L^{2}\left(\mathbb{R}^{d}\right) \mid\|f\|_{2}=1, \mathcal{E}_{\psi}(f, f)<\infty\right\} .
$$


and

$$
M_{\psi, p}=\sup _{f \in \mathcal{F}_{\psi}}\left\{\left(\int_{\mathbb{R}^{d}}|f(x)|^{2 p} \mathrm{~d} x\right)^{1 / p}-\mathcal{E}_{\psi}(f, f)\right\} .
$$

In the next section we show that $M_{\psi, p}<\infty$ when $p(d-\beta)<d$, and that $M_{\psi, p}$ can be expressed in terms of the best possible constant in a Gagliardo-Nirenberg type inequality.

We can now present our theorem describing the exponential asymptotics and large deviations for $\alpha_{p, t}$.

Theorem 1. Assume that $p(d-\beta)<d$. Then for any $\lambda>0$

$$
\lim _{t \rightarrow \infty} t^{-1} \log E\left(\mathrm{e}^{\lambda \alpha_{p, t}^{1 / p}}\right)=\lambda \frac{p \beta}{p \beta-d(p-1)} p^{-\frac{d(p-1)}{p \beta-d(p-1)}} M_{\psi, p} .
$$

Equivalently for any $h>0$

$$
\lim _{t \rightarrow \infty} t^{-1} \log P\left\{\alpha_{p, t}^{1 / p} \geqslant h t\right\}=-h^{p \beta / d(p-1)} A_{\psi, p}
$$

where

$$
A_{\psi, p}=\frac{d(p-1)}{\beta}\left(\frac{\beta p-d(p-1)}{\beta p M_{\psi, p}}\right)^{\frac{\beta p-d(p-1)}{d(p-1)}} .
$$

Using the scaling (1.7) our theorem is equivalent to the fact that for any $h>0$

$$
\lim _{t \rightarrow \infty} t^{-1} \log P\left\{\alpha_{p, 1}^{\beta / d(p-1)} \geqslant h t\right\}=-h A_{\psi, p} .
$$

Thus

$$
E\left(\mathrm{e}^{\lambda \alpha_{p, 1}^{\beta / d(p-1)}}\right) \begin{cases}<\infty & \text { if } \lambda<A_{\psi, p}^{-1}, \\ =\infty & \text { if } \lambda>A_{\psi, p}^{-1} .\end{cases}
$$

We next describe the law of the iterated logarithm for $\alpha_{p, t}$.

Theorem 2. Assume that $p(d-\beta)<d$. Then

$$
\limsup _{t \rightarrow \infty} t^{-(p-(p-1) d / \beta)}(\log \log t)^{-(p-1) d / \beta} \alpha_{p, t}=A_{\psi, p}^{-(p-1) d / \beta} \quad \text { a.s. }
$$

For the case of Brownian motion, i.e. $\beta=2$, these results were obtained by the first author in [3]. The methods of that paper depended heavily on the continuity of the Brownian path and the fact that the generator of Brownian motion, the Laplacian, is a local operator. In the course of overcoming the various problems associated with the stable process we have developed a new approach which greatly simplifies the proofs even for the case of Brownian motion.

We have also developed analogous results for random walks. Thus, consider $S_{1}(n), \ldots, S_{p}(n)$ independent copies of a $d$-dimensional symmetric random walk $S(n)$. We will assume that our random walks are in the domain of attraction of our nondegenerate symmetric stable process $X(t)$ of index $\beta$, i.e.

$$
\frac{S(n)}{b(n)} \rightarrow X(1)
$$

with $b(x)$ a function of regular variaton of index $1 / \beta$. Set

$$
I_{p, n}=\sum_{n_{1}, \ldots, n_{k}=0}^{n}\left[\sum_{x \in \mathbb{Z}^{d}} \prod_{j=1}^{p} \delta\left(S_{j}\left(n_{j}\right), x\right)\right]
$$


where

$$
\delta(y, x)= \begin{cases}1 & \text { if } x=y, \\ 0 & \text { otherwise }\end{cases}
$$

is the usual Kroenecker delta.

Let $\left\{v_{n}\right\}$ represents a positive sequence satisfying

$$
v_{n} \rightarrow \infty \text { and } v_{n} / n \rightarrow 0 .
$$

Here is our analogue of Theorem 1 describing the exponential asymptotics and moderate deviations for $I_{p, n}$.

Theorem 3. For any $\lambda>0$

$$
\lim _{n \rightarrow \infty} \frac{1}{v_{n}} \log E \exp \left\{\lambda \frac{v_{n}}{n} b\left(n v_{n}^{-1}\right)^{\frac{d(p-1)}{p}} I_{p, n}^{1 / p}\right\}=\lambda \frac{p \beta}{p \beta-d(p-1)} p^{-\frac{d(p-1)}{p \beta-d(p-1)}} M_{\psi, p}
$$

and for any $h>0$

$$
\lim _{n \rightarrow \infty} \frac{1}{v_{n}} \log P\left\{I_{n} \geqslant h n^{p} b\left(n v_{n}^{-1}\right)^{-d(p-1)}\right\}=-h^{\frac{\beta}{d(p-1)}} A_{\psi, p} .
$$

This gives rise to the following LIL for $I_{p, n}$.

\section{Theorem 4.}

$$
\limsup _{n \rightarrow \infty} n^{-p} b\left(\frac{n}{\log \log n}\right)^{d(p-1)} I_{p, n}=A_{\psi, p}^{-(p-1) d / \beta} \quad \text { a.s. }
$$

\section{Sobolev inequalities and Feynman-Kac formulae}

Lemma 1. If $p>1$ and $\beta>d(p-1) / p$ then $\mathcal{F}_{\psi} \subseteq L^{2 p}\left(\mathbb{R}^{d}\right)$, and for any $\delta>0$

$$
\|f\|_{2 p}^{2} \leqslant C_{\delta}\|f\|_{2}^{2}+\delta \mathcal{E}_{\psi}(f, f)
$$

for some $C_{\delta}<\infty$. In particular for any $\lambda>0$

$$
M_{\psi, p}(\lambda)=: \sup _{f \in \mathcal{F}_{\beta}}\left(\lambda\|f\|_{2 p}^{2}-\mathcal{E}_{\psi}(f, f)\right)<\infty .
$$

Proof of Lemma 1. When $\psi(\lambda)=|\lambda|^{\beta}$ we write $\mathcal{F}_{\beta}, \mathcal{E}_{\beta}, M_{\beta, p, d}$ for $\mathcal{F}_{\psi}, \mathcal{E}_{\psi}, M_{\psi, p}$. Because of (1.1) we have $\mathcal{E}_{\psi} \geqslant C \mathcal{E}_{\beta}$ and hence it suffices to prove (2.1) when $\psi(\lambda)=|\lambda|^{\beta}$. By the Hausdorff-Young inequality

$$
\|f\|_{2 p} \leqslant\|\hat{f}\|_{2 p /(2 p-1)}
$$

where $\hat{f}$ denotes the Fourier transform of $f$. We also have that for any $r>0$

$$
\begin{aligned}
\|\hat{f}\|_{2 p /(2 p-1)}^{2 p /(2 p-1)} & =\int_{\mathbb{R}^{d}} \frac{\left(r+|\lambda|^{\beta}\right)^{p /(2 p-1)}}{\left(r+|\lambda|^{\beta}\right)^{p /(2 p-1)}}|\hat{f}(\lambda)|^{2 p /(2 p-1)} \mathrm{d} \lambda \\
& \leqslant\left\|\left(r+|\lambda|^{\beta}\right)^{-p /(2 p-1)}\right\|_{(2 p-1) /(p-1)}\left\|\left(r+|\lambda|^{\beta}\right)^{p /(2 p-1)}|\hat{f}(\lambda)|^{2 p /(2 p-1)}\right\|_{(2 p-1) / p} .
\end{aligned}
$$

Now

$$
\left\|\left(r+|\lambda|^{\beta}\right)^{p /(2 p-1)}|\hat{f}(\lambda)|^{2 p /(2 p-1)}\right\|_{(2 p-1) / p}^{(2 p-1) / p}=r\|f\|_{2}^{2}+\mathcal{E}_{\beta}(f, f)
$$


and

$$
c_{r}=:\left\|\left(r+|\lambda|^{\beta}\right)^{-p /(2 p-1)}\right\|_{(2 p-1) /(p-1)}^{(2 p-1) /(p-1)}=\int_{\mathbb{R}^{d}} \frac{1}{\left(r+|\lambda|^{\beta}\right)^{p /(p-1)}} \mathrm{d} \lambda
$$

which is finite if $\beta>d(p-1) / p$, in which case we also have that $\lim _{r \rightarrow \infty} c_{r}=0$. Summarizing,

$$
\|f\|_{2 p}^{2} \leqslant c_{r}^{(p-1) / p}\left(r\|f\|_{2}^{2}+\mathcal{E}_{\beta}(f, f)\right) .
$$

This gives (2.1) on taking $r$ sufficiently large. This completes the proof of our lemma.

Set $M_{\psi, p}=M_{\psi, p}(1)$

Lemma 2. If $p>1$ and $\beta>d(p-1) / p$ then

$$
\kappa_{\psi, p}=: \inf \left\{C \mid\|f\|_{2 p} \leqslant C\|f\|_{2}^{1-d(p-1) / p \beta}\left[\mathcal{E}_{\psi}^{1 / 2}(f, f)\right]^{d(p-1) / p \beta}, \forall f \in \mathcal{F}_{\psi}\right\}<\infty
$$

and

$$
M_{\psi, p}(1)=\frac{p \beta-d(p-1)}{d(p-1)}\left(\frac{d(p-1) \kappa_{\psi, p}^{2}}{p \beta}\right)^{p \beta /(p \beta-d(p-1))} .
$$

For any $\lambda>0$

$$
M_{\psi, p}(\lambda)=\lambda^{p \beta /(p \beta-d(p-1))} M_{\psi, p} .
$$

Proof of Lemma 2. To see that (2.8) is finite, note that if we set $f(x)=s^{d / 2} g(s x)$, then $\|f\|_{2}=\|g\|_{2},\|f\|_{2 p}^{2}=$ $s^{d(p-1) / p}\|g\|_{2 p}^{2}$ and $\mathcal{E}_{\psi}(f, f)=s^{\beta} \mathcal{E}_{\psi}(g, g)$ so that from (2.1) we obtain

$$
\|g\|_{2 p}^{2} \leqslant C\left(\|g\|_{2}^{2}+s^{\beta} \mathcal{E}_{\beta}(g, g)\right) s^{-d(p-1) / p}
$$

and the fact that (2.8) is finite follows on taking $s^{\beta}=\|g\|_{2}^{2} / \mathcal{E}_{\beta}(g, g)$. The same scaling establishes (2.10). Finally, (2.9) follows as in the proof of Lemma 8.2 of [3]. This completes the proof of our lemma.

\section{Large deviations}

In this section we show how to obtain our large deviation result for the intersection local time, Theorem 1, from a large deviation result for an approximate intersection local time together with exponential approximation.

Let $X_{1}(t), \ldots, X_{p}(t)$ be independent $d$-dimensional symmetric stable processes of index $\beta$. We assume $p(d-\beta)<d$. Recall that the approximate intersection local time is defined by

$$
\alpha_{p, t, \epsilon}=\int_{0}^{t} \cdots \int_{0}^{t}\left[\int_{\mathbb{R}^{d}} \prod_{j=1}^{p} h_{\epsilon}\left(X_{j}\left(s_{j}\right)-x\right) \mathrm{d} x\right] \mathrm{d} s_{1} \cdots \mathrm{d} s_{p}
$$

and that

$$
\alpha_{p, t}=\lim _{\epsilon \rightarrow 0} \alpha_{p, t, \epsilon}
$$

The following large deviation result for $\alpha_{p, t, \epsilon}$ will be proven in Section 4. 
Theorem 5. Assume that $p(d-\beta)<d$. Then for any $\lambda>0$

$$
\lim _{t \rightarrow \infty} \frac{1}{t} \log E \exp \left\{\lambda \alpha_{\epsilon, t}^{1 / p}\right\}=\sup _{g \in \mathcal{F}_{\psi}}\left\{\lambda\left(\int_{\mathbb{R}^{d}}\left|\left(g^{2} * h_{\epsilon}\right)(x)\right|^{p}\right)^{1 / p}-p \mathcal{E}_{\psi}(g, g)\right\} .
$$

The following theorem on exponential approximation will be proven in Section 6 .

Theorem 6. Assume that $p(d-\beta)<d$. Then for any $\lambda>0$,

$$
\limsup _{\epsilon \rightarrow 0} \limsup _{t \rightarrow \infty} \frac{1}{t} \log E \exp \left\{\lambda\left|\alpha_{p, t, \epsilon}-\alpha_{p, t}\right|^{1 / p}\right\}=0 .
$$

Proof of Theorem 1. By Hölder's inequality,

$$
E \exp \left\{\lambda a^{-1} \alpha_{p, t, \epsilon}^{1 / p}\right\} \leqslant\left(E \exp \left\{\lambda \alpha_{p, t}^{1 / p}\right\}\right)^{1 / a}\left(E \exp \left\{b a^{-1} \lambda\left|\alpha_{p, t, \epsilon}-\alpha_{p, t}\right|^{1 / p}\right\}\right)^{1 / b}
$$

where $a, b>1$ and $a^{-1}+b^{-1}=1$. Hence by Theorem 5

$$
\begin{aligned}
& \sup _{g \in \mathcal{F}_{\beta}}\left\{\lambda a^{-1}\left(\int_{\mathbb{R}^{d}}\left|\left(g^{2} * h_{\epsilon}\right)(x)\right|^{p}\right)^{1 / p}-p \mathcal{E}_{\beta}(g, g)\right\} \\
& \quad \leqslant \liminf _{t \rightarrow \infty} \frac{1}{a t} \log E \exp \left\{\lambda \alpha_{p, t}^{1 / p}\right\}+\limsup _{t \rightarrow \infty} \frac{1}{b t} \log E \exp \left\{b a^{-1} \lambda\left|\alpha_{p, t, \epsilon}-\alpha_{p, t}\right|^{1 / p}\right\} .
\end{aligned}
$$

Letting $\epsilon \rightarrow 0$ and using Theorem 6 and (2.10)

$$
\begin{aligned}
\liminf _{t \rightarrow \infty} \frac{1}{t} \log E \exp \left\{\lambda \alpha_{p, t}^{1 / p}\right\} & \geqslant a \sup _{g \in \mathcal{F}_{\psi}}\left\{\lambda a^{-1}\left(\int_{\mathbb{R}^{d}}|g(x)|^{2 p} \mathrm{~d} x\right)^{1 / p}-p \mathcal{E}_{\psi}(g, g)\right\} \\
& =a p^{-\frac{d(p-1)}{p \beta-d(p-1)}}\left(\lambda a^{-1}\right)^{\frac{p \beta}{p \beta-d(p-1)}} M_{\psi, p} .
\end{aligned}
$$

Letting $a \rightarrow 1$,

$$
\liminf _{t \rightarrow \infty} \frac{1}{t} \log E \exp \left\{\lambda \alpha_{p, t}^{1 / p}\right\} \geqslant p^{-\frac{d(p-1)}{p \beta-d(p-1)}} \lambda \frac{p \beta}{p \beta-d(p-1)} M_{\psi, p} .
$$

On the other hand,

$$
E \exp \left\{\lambda \alpha_{p, t}^{1 / p}\right\} \leqslant\left(E \exp \left\{a \lambda \alpha_{p, t, \epsilon}^{1 / p}\right\}\right)^{1 / a}\left(E \exp \left\{b \lambda\left|\alpha_{p, t, \epsilon}-\alpha_{p, t}\right|^{1 / p}\right\}\right)^{1 / b} .
$$

Therefore, using Theorem 5

$$
\begin{aligned}
& \limsup _{t \rightarrow \infty} \frac{1}{t} \log E \exp \left\{\lambda \alpha_{p, t}^{1 / p}\right\} \\
& \quad \leqslant a^{-1} \sup _{g \in \mathcal{F}_{\psi}}\left\{a \lambda\left(\int_{\mathbb{R}^{d}}\left|\left(g^{2} * h_{\epsilon}\right)(x)\right|^{p} \mathrm{~d} x\right)^{1 / p}-p \mathcal{E}_{\psi}(g, g)\right\}+\limsup _{t \rightarrow \infty} \frac{1}{b t} \log E \exp \left\{b \lambda\left|\alpha_{p, t, \epsilon}-\alpha_{t}\right|^{1 / p}\right\} \\
& \quad \leqslant a^{-1} \sup _{g \in \mathcal{F}_{\psi}}\left\{a \lambda\left(\int_{\mathbb{R}^{d}}|g(x)|^{2 p} \mathrm{~d} x\right)^{1 / p}-p \mathcal{E}_{\psi}(g, g)\right\}+\limsup _{t \rightarrow \infty} \frac{1}{b t} \log E \exp \left\{b \lambda\left|\alpha_{p, t, \epsilon}-\alpha_{p, t}\right|^{1 / p}\right\} .
\end{aligned}
$$

Letting $\epsilon \rightarrow 0$ and using Theorem 6 and (2.10) 


$$
\begin{aligned}
\limsup _{t \rightarrow \infty} \frac{1}{t} \log E \exp \left\{\lambda \alpha_{p, t}^{1 / p}\right\} & \leqslant a^{-1} \sup _{g \in \mathcal{F}_{\psi}}\left\{a \lambda\left(\int_{\mathbb{R}^{d}}|g(x)|^{2 p} \mathrm{~d} x\right)^{1 / p}-p \mathcal{E}_{\psi}(g, g)\right\} \\
& =a^{-1} p^{-\frac{d(p-1)}{p \beta-d(p-1)}}(\lambda a)^{\frac{p \beta}{p \beta-d(p-1)}} M_{\psi, p} .
\end{aligned}
$$

Letting $a \rightarrow 1$,

$$
\limsup _{t \rightarrow \infty} \frac{1}{t} \log E \exp \left\{\lambda \alpha_{p, t}^{1 / p}\right\} \leqslant p^{-\frac{d(p-1)}{p \beta-d(p-1)}} \lambda^{\frac{p \beta}{p \beta-d(p-1)}} M_{\psi, p} .
$$

Combining what we have,

$$
\lim _{t \rightarrow \infty} \frac{1}{t} \log E \exp \left\{\lambda \alpha_{p, t}^{1 / p}\right\}=p^{-\frac{d(p-1)}{p \beta-d(p-1)}} \lambda \frac{p \beta}{p \beta-d(p-1)} M_{\psi, p}, \quad \lambda>0 .
$$

Finally, by the Gärtner-Ellis theorem, Theorem 2.3.6 of [6],

$$
\begin{aligned}
\lim _{t \rightarrow \infty} \frac{1}{t} \log P\left\{\alpha_{p, t}^{1 / p} \geqslant h t\right\} & =-\sup _{\lambda>0}\left\{\lambda h-p^{-\frac{d(p-1)}{p \beta-d(p-1)}} \lambda \frac{p \beta}{p \beta-d(p-1)} M_{\psi, p}\right\} \\
& =-h^{p \beta / d(p-1)} \frac{d(p-1)}{\beta}\left(\frac{\beta p-d(p-1)}{\beta p M_{\psi, p}}\right)^{\frac{\beta p-d(p-1)}{d(p-1)}} .
\end{aligned}
$$

\section{Exponential asymptotics for the approximate intersection local time}

In this section, we fix $\epsilon>0$ and write

$$
L(t, x, \epsilon)=\int_{0}^{t} h_{\epsilon}(X(s)-x) \mathrm{d} s \quad x \in \mathbb{R}^{d}, t \geqslant 0 .
$$

For each $1 \leqslant j \leqslant p$, let $L_{j}(t, x, \epsilon)$ be the analogues of $L(t, x, \epsilon)$ with $X(t)$ being replaced by $X_{j}(t)$.

For any $\theta>0$, using $*$ to denote convolution, write

$$
\begin{aligned}
& M_{\psi, p, \epsilon}(\theta)=\sup _{f \in \mathcal{F}_{\psi}}\left\{\theta\left(\int_{\mathbb{R}^{d}}\left[f^{2} * h_{\epsilon}(x)\right]^{p} \mathrm{~d} x\right)^{1 / p}-\mathcal{E}_{\psi}(f, f)\right\}, \\
& N_{\psi, p, \epsilon}(\theta)=\sup _{f \in \mathcal{F}_{\psi}}\left\{\theta\left(\int_{\mathbb{R}^{d}}\left[f^{2} * h_{\epsilon}(x)\right]^{p} \mathrm{~d} x\right)^{1 / p}-p \mathcal{E}_{\psi}(f, f)\right\} .
\end{aligned}
$$

By the fact that $\|f\|_{2}=1$

$$
\int_{\mathbb{R}^{d}}\left[f^{2} * h_{\epsilon}(x)\right]^{p} \mathrm{~d} x \leqslant \sup _{x \in R^{d}}\left[f^{2} * h_{\epsilon}(x)\right]^{p-1} \leqslant\left(\frac{\|h\|_{\infty}}{\epsilon^{d}}\right)^{p-1}
$$

so that the functions $M_{\psi, p, \epsilon}(\cdot)$ and $N_{\psi, p, \epsilon}(\cdot)$ are continuous for any fixed $\epsilon>0$.

Theorem 7. For any $\theta>0$ and integers $d \geqslant 1, p \geqslant 2$,

$$
\lim _{t \rightarrow \infty} \frac{1}{t} \log E \exp \left\{\theta\left(\int_{\mathbb{R}^{d}} L^{p}(t, x, \epsilon) \mathrm{d} x\right)^{1 / p}\right\}=M_{\psi, p, \epsilon}(\theta),
$$


and

$$
\lim _{t \rightarrow \infty} \frac{1}{t} \log E \exp \left\{\theta\left(\int_{\mathbb{R}^{d}} \prod_{j=1}^{p} L_{j}(t, x, \epsilon) \mathrm{d} x\right)^{1 / p}\right\}=N_{\psi, p, \epsilon}(\theta) .
$$

Proof of Theorem 7. We start with the following result based on the Feynman-Kac formula:

$$
\lim _{t \rightarrow \infty} \frac{1}{t} \log E \exp \left\{\int_{0}^{t} f(X(s)) \mathrm{d} s\right\} \geqslant \sup _{g \in \mathcal{F}_{\psi}}\left\{\int_{\mathbb{R}^{d}} f(x) g^{2}(x) \mathrm{d} x-\mathcal{E}_{\psi}(g, g)\right\}
$$

where $f$ can be any bounded, measurable function $f$ on $\mathbb{R}^{d}$. This can be proven in a manner similar to our proof of (4.2) in [5], which deals with the one-dimensional case. (Alternatively, (4.6) can be derived by the methods used in [7], which also deals with the one-dimensional case. Using those methods one can show that we have equality in (4.6), although we will not need that.)

We begin by proving the lower bounds for (4.4) and (4.5). Notice that for any $r>0$, and any measurable function $f$ on $R^{d}$ with $|f|_{q}=1$ and $f(x)=0$ for $|x|>r$

$$
\left(\int_{\{|x| \leqslant r\}} L^{p}(t, x, \epsilon) \mathrm{d} x\right)^{1 / p} \geqslant \int_{\mathbb{R}^{d}} f(x) L(t, x, \epsilon) \mathrm{d} x=\int_{0}^{t} f * h_{\epsilon}(X(s)) \mathrm{d} s .
$$

By (4.6) we have

$$
\begin{aligned}
& \liminf _{t \rightarrow \infty} \frac{1}{t} \log E \exp \left\{\theta\left(\int_{\{|x| \leqslant r\}} L^{p}(t, x, \epsilon) \mathrm{d} x\right)^{1 / p}\right\} \\
& \geqslant \sup _{g \in \mathcal{F}_{\psi}}\left\{\theta \int_{\mathbb{R}^{d}} f * h_{\epsilon}(x) g^{2}(x) \mathrm{d} x-\mathcal{E}_{\psi}(g, g)\right\} \\
& \quad=\sup _{g \in \mathcal{F}_{\psi}}\left\{\theta \int_{\{|x| \leqslant r\}} f(x) g^{2} * h_{\epsilon}(x) \mathrm{d} x-\mathcal{E}_{\psi}(g, g)\right\} .
\end{aligned}
$$

Taking the supremum over $f$ on the right-hand side,

$$
\begin{aligned}
& \liminf _{t \rightarrow \infty} \frac{1}{t} \log E \exp \left\{\theta\left(\int_{\{|x| \leqslant r\}} L^{p}(t, x, \epsilon) \mathrm{d} x\right)^{1 / p}\right\} \\
& \geqslant \sup _{g \in \mathcal{F}_{\psi}}\left\{\theta\left(\int_{\{|x| \leqslant r\}}\left|g^{2} * h_{\epsilon}(x)\right|^{p} \mathrm{~d} x\right)^{1 / p}-\mathcal{E}_{\psi}(g, g)\right\} .
\end{aligned}
$$

In particular, letting $r=\infty$ gives the lower bound for (4.4).

To prove the lower bound for (4.5), we let $r>0$ be finite in (4.9). For any function $f(x)$, let $\mathcal{R}_{r} f(x)$ be the restriction of $f(x)$ to $B_{r}$, the closed ball of radius $r$ centered at the origin. It follows from the definition (4.1) that $\|L(t, \cdot, \epsilon)\|_{\infty} \leqslant\left\|h_{\epsilon}\right\|_{\infty} t$ and $|L(t, x, \epsilon)-L(t, y, \epsilon)| \leqslant\left\|\nabla h_{\epsilon}\right\|_{\infty} t|x-y|$ for all $x, y$. Hence if we set

$$
A_{r, \epsilon}=\left\{f \in C\left(B_{r}\right)|| f(x) \mid \leqslant\left\|h_{\epsilon}\right\|_{\infty}, \forall x \in B_{r} \text { and }|f(x)-f(y)| \leqslant\left\|\nabla h_{\epsilon}\right\|_{\infty}|x-y|, \forall x, y \in B_{r}\right\}
$$

we have that

$$
\frac{1}{t} \mathcal{R}_{r} L(t, \cdot, \epsilon) \in A_{r, \epsilon}
$$


Note that by Ascoli's Lemma $A_{r, \epsilon}$ is a precompact subset of $C\left(B_{r}\right)$ in the uniform norm, and a fortiori $A_{r, \epsilon}$ is a precompact subset of $L^{p}\left(B_{r}\right)$. We use $K_{r, \epsilon}$ to denote the closure of $A_{r, \epsilon}$ in $L^{p}\left(B_{r}\right)$.

Consider the continuous, non-negative functional $\Psi$ defined on $\left(L^{p}\left(B_{r}\right)\right)^{p}$ :

$$
\Psi\left(f_{1}, \ldots, f_{p}\right)=\frac{1}{p} \sum_{j=1}^{p}\left(\int_{\{|x| \leqslant r\}}\left|f_{j}(x)\right|^{p} \mathrm{~d} x\right)^{1 / p}-\left(\int_{\{|x| \leqslant r\}} \prod_{j=1}^{p}\left|f_{j}(x)\right| \mathrm{d} x\right)^{1 / p} .
$$

Clearly, $\Psi \equiv 0$ on the diagonal

$$
\left\{\left(f_{1}, \ldots, f_{p}\right) ; f_{1}=\cdots=f_{p}\right\} .
$$

Hence, for given $\delta>0$ and any $g \in L^{p}\left(B_{r}\right)$ there exists a $b=b(g, \delta)>0$ such that

$$
\Psi\left(f_{1}, \ldots, f_{p}\right) \leqslant \delta \quad \text { if } f_{j} \in B(g, b) \text { for } \forall 1 \leqslant j \leqslant p
$$

where $B(g, b)$ stands for the open ball in $L^{p}\left(B_{r}\right)$ with the center $g$ and the radius $b$. Therefore,

$$
\begin{aligned}
& E \exp \left\{\theta\left(\int_{\{|x| \leqslant r\}} \prod_{j=1}^{p} L_{j}(t, x, \epsilon) \mathrm{d} x\right)^{1 / p}\right\} \\
& \geqslant \mathrm{e}^{-\delta t} E\left[\exp \left\{\frac{\theta}{p} \sum_{j=1}^{p}\left(\int_{\{|x| \leqslant r\}} L_{j}^{p}(t, x, \epsilon) \mathrm{d} x\right)^{1 / p}\right\} ; \frac{1}{t} \mathcal{R}_{r} L_{j}(t, \cdot, \epsilon) \in B(g, b), \forall 1 \leqslant j \leqslant p\right] \\
& =\mathrm{e}^{-\delta t}\left(E\left[\exp \left\{\frac{\theta}{p}\left(\int_{\{|x| \leqslant r\}} L^{p}(t, x, \epsilon) \mathrm{d} x\right)^{1 / p}\right\} ; \frac{1}{t} \mathcal{R}_{r} L(t, \cdot, \epsilon) \in B(g, b)\right]\right)^{p} .
\end{aligned}
$$

Let $\left\{B\left(g_{1}, b_{1}\right), \ldots, B\left(g_{N}, b_{N}\right)\right\}$ be a finite sub-family of the open sets

$$
\left\{B(g, b(g, \delta)) ; g \in K_{r, \epsilon}\right\}
$$

which covers $K_{r, \epsilon}$. Then by (4.11)

$$
\begin{aligned}
& E\left[\exp \left\{\frac{\theta}{p}\left(\int_{\{|x| \leqslant r\}} L^{p}(t, x, \epsilon) \mathrm{d} x\right)^{1 / p}\right\}\right] \\
& \quad \leqslant \sum_{i=1}^{N} E\left[\exp \left\{\frac{\theta}{p}\left(\int_{B_{r}} L^{p}(t, x, \epsilon) \mathrm{d} x\right)^{1 / p}\right\} ; \frac{1}{t} \mathcal{R}_{r} L(t, \cdot, \epsilon) \in B\left(g_{i}, b_{i}\right)\right] .
\end{aligned}
$$

Therefore,

$$
\begin{aligned}
& \liminf _{t \rightarrow \infty} \frac{1}{t} \log \max _{1 \leqslant i \leqslant N} E\left[\exp \left\{\frac{\theta}{p}\left(\int_{\{|x| \leqslant r\}} L^{p}(t, x, \epsilon) \mathrm{d} x\right)^{1 / p}\right\} ; \frac{1}{t} \mathcal{R}_{r} L(t, \cdot, \epsilon) \in B\left(g_{i}, b_{i}\right)\right] \\
& \left.\geqslant \liminf _{t \rightarrow \infty} \frac{1}{t} \log E\left[\exp \left\{\frac{\theta}{p} \int_{\{|x| \leqslant r\}} L^{p}(t, x, \epsilon) \mathrm{d} x\right)^{1 / p}\right\}\right] .
\end{aligned}
$$

Combining this with (4.9), (with $\theta$ being replaced by $p^{-1} \theta$ ), and with (4.14) we have 


$$
\begin{aligned}
& \liminf _{t \rightarrow \infty} \frac{1}{t} \log E \exp \left\{\theta\left(\int_{\{|x| \leqslant r\}} \prod_{j=1}^{p} L_{j}(t, x, \epsilon) \mathrm{d} x\right)^{1 / p}\right\} \\
& \geqslant-\delta+p \sup _{g \in \mathcal{F}_{\psi}}\left\{\frac{\theta}{p}\left(\int_{\{|x| \leqslant r\}}\left|g^{2} * h_{\epsilon}(x)\right|^{p} \mathrm{~d} x\right)^{1 / p}-\mathcal{E}_{\psi}(g, g)\right\} .
\end{aligned}
$$

Letting $\delta \rightarrow 0^{+}$and $r \rightarrow \infty$ we obtain the lower bound for (4.5):

$$
\liminf _{t \rightarrow \infty} \frac{1}{t} \log E \exp \left\{\theta\left(\int_{\mathbb{R}^{d}} \prod_{j=1}^{p} L_{j}(t, x, \epsilon) \mathrm{d} x\right)^{1 / p}\right\} \geqslant N_{\psi, p, \epsilon}(\theta) .
$$

We now prove the upper bound for (4.4). We may let $t \rightarrow \infty$ along the integers. Let $m>0$ be fixed and let $G_{m}$ be the discrete subgroup of $R^{d}$ consisting of vectors whose coordinates are integer multiples of $m$. Let $T_{m}^{d}$ be the quotient of $\mathbb{R}^{d}$ modulo $G_{m}$ and let $\iota: \mathbb{R}^{d} \rightarrow T_{m}^{d}$ be the canonical map. Then the $T_{m}^{d}$-valued process $X_{*}(t)=\iota(X(t))$ is Markov process, the symmetric stable process on the torus $T_{m}^{d}$. Notice that $T_{m}^{d}$ becomes a compact group under the induced distance $d\left(x_{*}, y_{*}\right)=|x-y|$, where $x$ and $y$ satisfy $\iota(x)=x_{*}, \iota(y)=y_{*}$ and $x, y \in[0, m)^{d}$. Let $\lambda\left(d x_{*}\right)$ be the Lebesgue (Haar) measure on $T_{m}^{d}$ and write

$$
L_{*}\left(t, x_{*}, \epsilon\right)=\sum_{\mathbf{k} \in \mathbb{Z}^{d}} L(t, x+m \mathbf{k}, \epsilon)=\int_{0}^{t} h_{\epsilon *}\left(X_{*}(s)-x_{*}\right) \mathrm{d} s, \quad t \geqslant 0, x_{*} \in T_{m}^{d}
$$

where $h_{\epsilon *}$ is a function on $T_{m}^{d}$ defined by

$$
h_{\epsilon *}\left(x_{*}\right)=\sum_{\mathbf{k} \in \mathbb{Z}^{d}} h_{\epsilon}(x+m \mathbf{k}) .
$$

Notice that

$$
\begin{aligned}
\int_{\mathbb{R}^{d}} L^{p}(t, x, \epsilon) \mathrm{d} x & =\sum_{\mathbf{k} \in \mathbb{Z}^{d}[0, m]^{d}} \int_{[0, m]^{d}} L^{p}(t, x+m \mathbf{k}, \epsilon) \mathrm{d} x \\
& \left.\leqslant \int_{\mathbf{k} \in \mathbb{Z}^{d}} L(t, x+m \mathbf{k}, \epsilon)\right]^{p} \mathrm{~d} x=\int_{\mathbb{T}_{m}^{d}}\left[L_{*}\left(t, x_{*}, \epsilon\right)\right]^{p} \lambda\left(\mathrm{d} x_{*}\right) .
\end{aligned}
$$

Using the methods we used in the proof of Lemma 6 of [5], which deals with the one-dimensional case, we can show that for any bounded, measurable function $f$ on $T_{m}^{d}$

$$
\lim _{t \rightarrow \infty} \frac{1}{t} \log E \exp \left\{\int_{0}^{t} f\left(X_{*}(s)\right) \mathrm{d} s\right\}=\sup _{g \in \mathcal{F}_{\psi, T_{m}^{d}}}\left\{\int_{T_{m}^{d}} f(x) g^{2}(x) \lambda(\mathrm{d} x)-\mathcal{E}_{\psi, T_{m}^{d}}(g, g)\right\} .
$$

Here

$$
\mathcal{E}_{\psi, T_{m}^{d}}(g, g)=: \sum_{\lambda \in\left(\frac{2 \pi}{m}\right) \mathbb{Z}^{d}} \psi(\lambda)|\hat{g}(\lambda)|^{2} \frac{1}{m^{d}}
$$

where $\hat{g}(\lambda)$ denotes the Fourier transform of $g \in L^{2}\left(T_{m}^{d}\right)$, and

$$
\mathcal{F}_{\psi, T_{m}^{d}}=\left\{g \in L^{2}\left(T_{m}^{d}\right) \mid\|g\|_{2, T_{m}^{d}}=1, \mathcal{E}_{\psi, T_{m}^{d}}(g, g)<\infty\right\} .
$$


We will use the notation $f *_{m} g(x)=\int_{T_{m}^{d}} f(x-y) g(y) \lambda(\mathrm{d} y)$ for convolution of functions on $T_{m}^{d}$. By (4.22),

$$
\begin{aligned}
& \lim _{t \rightarrow \infty} \frac{1}{t} \log E \exp \left\{\int_{T_{m}^{d}} f\left(x_{*}\right) L_{*}\left(t, x_{*}, \epsilon\right) \lambda\left(\mathrm{d} x_{*}\right)\right\} \\
& =\lim _{t \rightarrow \infty} \frac{1}{t} \log E \exp \left\{\int_{0}^{t} f *_{m} h_{\epsilon, *}\left(X_{*}(s)\right) \mathrm{d} s\right\} \\
& =\sup _{g \in \mathcal{F}_{\psi, T_{m}^{d}}}\left\{\int_{T_{m}^{d}} f *_{m} h_{\epsilon, *}(x) g^{2}(x) \lambda(\mathrm{d} x)-\mathcal{E}_{\psi, T_{m}^{d}}(g, g)\right\} \\
& =\sup _{g \in \mathcal{F}_{\psi, T_{m}^{d}}}\left\{\int_{T_{m}^{d}} f(x) g^{2} *_{m} h_{\epsilon, *}(x) \lambda(\mathrm{d} x)-\mathcal{E}_{\psi, T_{m}^{d}}(g, g)\right\} .
\end{aligned}
$$

From (4.20) and the fact that $h_{\epsilon} \in \mathcal{S}\left(\mathbb{R}^{d}\right)$ we see that $\left\|h_{\epsilon *}\right\|_{\infty}$ and $\left\|\nabla h_{\epsilon *}\right\|_{\infty}$ are both finite. It follows from the definitions (4.19) that $\left\|L_{*}(t, \cdot, \epsilon)\right\|_{\infty} \leqslant\left\|h_{\epsilon *}\right\|_{\infty} t$ and $\left|L_{*}(t, x, \epsilon)-L_{*}(t, y, \epsilon)\right| \leqslant\left\|\nabla h_{\epsilon *}\right\|_{\infty} t|x-y|$ for all $x, y$. Hence if we set

$$
\begin{aligned}
& A_{*, r, \epsilon}=\left\{f \in C\left(T_{m}^{d}\right)|| f(x) \mid \leqslant\left\|h_{\epsilon *}\right\|_{\infty}, \forall x \in T_{m}^{d} \text { and }|f(x)-f(y)| \leqslant\left\|\nabla h_{\epsilon *}\right\|_{\infty}|x-y|\right\}, \\
& \quad \forall x, y \in T_{m}^{d}
\end{aligned}
$$

we have that

$$
\frac{1}{t} L_{*}(t, \cdot, \epsilon) \in A_{*, r, \epsilon}
$$

Note as before that by Ascoli's Lemma $A_{*, r, \epsilon}$ is a precompact subset of $C\left(T_{m}^{d}\right)$ in the uniform norm, and a fortiori $A_{*, r, \epsilon}$ is a precompact subset of $L^{p}\left(T_{m}^{d}\right)$. We use $K_{*, r, \epsilon}$ to denote the closure of $A_{*, r, \epsilon}$ in $L^{p}\left(T_{m}^{d}\right)$. Let $q>1$ be the conjugate of $p$ and let $\delta>0$ be fixed. By the Hahn-Banach Theorem and compactness, there are finitely many bounded functions $f_{1}, \ldots, f_{N}$ in the unit sphere of $L^{q}\left(T_{M}\right)$ such that

$$
\left(\int_{T_{m}^{d}}|h(x)|^{p} \lambda(\mathrm{d} x)\right)^{1 / p}<\max _{1 \leqslant i \leqslant N} \int_{T_{m}^{d}} f_{i}(x) h(x) \lambda(\mathrm{d} x)+\delta \quad \forall h \in K_{*, r, \epsilon} .
$$

In particular, using (4.27)

$$
E\left(\exp \left\{\theta\left(\int_{T_{m}^{d}}\left[L_{*}(t, x, \epsilon)\right]^{p} \lambda(\mathrm{d} x)\right)^{1 / p}\right\}\right) \leqslant \mathrm{e}^{\delta t} \sum_{i=1}^{N} E \exp \left\{\theta \int_{T_{m}^{d}} f_{i}(x) L_{*}(t, x, \epsilon) \lambda(\mathrm{d} x)\right\} .
$$

Hence by (4.25),

$$
\begin{aligned}
& \limsup _{t \rightarrow \infty} \frac{1}{t} \log E \exp \left\{\theta\left(\int_{T_{m}^{d}}\left[L_{*}(t, x, \epsilon)\right]^{p} \lambda(\mathrm{d} x)\right)^{1 / p}\right\} \\
& \leqslant \delta+\max _{1 \leqslant i \leqslant N} \sup _{g \in \mathcal{F}_{\psi, T_{m}^{d}}}\left\{\theta \int_{T_{m}^{d}} f_{i}(x) g^{2} *_{m} h_{\epsilon, *}(x) \lambda(\mathrm{d} x)-\mathcal{E}_{\psi, T_{m}^{d}}(g, g)\right\} \\
& \leqslant \delta+\sup _{g \in \mathcal{F}_{\psi, T_{m}^{d}}}\left\{\theta\left(\int_{T_{m}^{d}}\left(g^{2} *_{m} h_{\epsilon, *}(x)\right)^{p} \mathrm{~d} x\right)^{1 / p}-\mathcal{E}_{\psi, T_{m}^{d}}(g, g)\right\} .
\end{aligned}
$$


In view of the relation (4.21) and Lemma 3 below, letting $\delta \rightarrow 0$ and then $m \rightarrow \infty$ we obtain the upper bound for (4.4):

$$
\limsup _{t \rightarrow \infty} \frac{1}{t} \log E \exp \left\{\theta\left(\int_{\mathbb{R}^{d}} L^{p}(t, x, \epsilon) \mathrm{d} x\right)^{1 / p}\right\} \leqslant M_{\psi, p, \epsilon}(\theta) .
$$

By the inequality

$$
\left(\int_{\mathbb{R}^{d}} \prod_{j=1}^{p} L_{j}(t, x, \epsilon) \mathrm{d} x\right)^{1 / p} \leqslant \frac{1}{p} \sum_{j=1}^{p}\left(\int_{\mathbb{R}^{d}} L_{j}^{p}(t, x, \epsilon) \mathrm{d} x\right)^{1 / p}
$$

we have

$$
E \exp \left\{\theta\left(\int_{\mathbb{R}^{d}} \prod_{j=1}^{p} L_{j}(t, x, \epsilon) \mathrm{d} x\right)^{1 / p}\right\} \leqslant\left[E \exp \left\{\frac{\theta}{p}\left(\int_{\mathbb{R}^{d}} L^{p}(t, x, \epsilon) \mathrm{d} x\right)^{1 / p}\right\}\right]^{p} .
$$

From (4.31), (with $\theta$ being replaced by $p^{-1} \theta$ ), we have the upper bound for (4.5):

$$
\limsup _{t \rightarrow \infty} \frac{1}{t} \log E \exp \left\{\theta\left(\int_{\mathbb{R}^{d}} \prod_{j=1}^{p} L_{j}(t, x, \epsilon) \mathrm{d} x\right)^{1 / p}\right\} \leqslant N_{\psi, p, \epsilon}(\theta) .
$$

\section{Localization}

In this section we assume $\beta<2$. The case of Brownian motion was developed in [3]. By the Lévy-Khintchine formula

$$
\psi(\lambda)=2 \int_{\mathbb{R}^{d}} \frac{1-\cos (\lambda \cdot y)}{J_{d+\beta}(y)} \mathrm{d} y
$$

where $J_{d+\beta}(y) \geqslant 0$ is a symmetric positively homogeneous function of degree $d+\beta$ and we may assume that for some $0<c<C<\infty$

$$
c|y|^{d+\beta} \leqslant J_{d+\beta}(y) \leqslant C|y|^{d+\beta} .
$$

Using Parseval's formula we find that

$$
\mathcal{E}_{\psi}(f, f)=\iint_{\mathbb{R}^{d}} \frac{|f(y)-f(x)|^{2}}{J_{d+\beta}(y-x)} \mathrm{d} y \mathrm{~d} x
$$

Recall that for a function $h(x)$ on $\mathbb{R}^{d}$ we set $h_{*}(x)=\sum_{\mathbf{k} \in \mathbb{Z}^{d}} h(x+m \mathbf{k})$.

Lemma 3. Let $p>1$ and let $h$ be any non-negative measurable function satisfying

$$
|h(x)| \leqslant \frac{C}{1+|x|^{d+\zeta}}
$$

for some $\zeta>0$. Then for any $\theta>0$ 


$$
\begin{aligned}
& \limsup \sup _{m \rightarrow \infty}\left\{\theta\left(\int_{\bar{g} \in \mathcal{F}_{\psi, T_{m}^{d}}}\left(\bar{g}^{2} *_{m} h_{*}(x)\right)^{p} \mathrm{~d} x\right)^{1 / p}-\mathcal{E}_{\psi, T_{m}^{d}}(\bar{g}, \bar{g})\right\} \\
& \quad \leqslant \sup _{g \in \mathcal{F}_{\psi}}\left\{\theta\left(\int_{\mathbb{R}^{d}}\left(g^{2} * h(x)\right)^{p} \mathrm{~d} x\right)^{1 / p}-\mathcal{E}_{\psi}(g, g)\right\} .
\end{aligned}
$$

Proof of Lemma 3. Let $\bar{g} \in \mathcal{F}_{\psi, T_{m}^{d}}$ be fixed. We may consider $\bar{g}$ to be extended to $\mathbb{R}^{d}$ by periodicity. Then

$$
\int_{[0, m]^{d}} \bar{g}^{2}(x) \mathrm{d} x=1 .
$$

and

$$
\bar{g}^{2} *_{m} h_{*}(x)=\int_{[0, m]^{d}} \bar{g}^{2}(x-y) \sum_{\mathbf{k} \in \mathbb{Z}^{d}} h(y+m \mathbf{k}) \mathrm{d} y=\bar{g}^{2} * h(x) .
$$

Hence

$$
\sup _{x \in[0, m]^{d}} \bar{g}^{2} * h(x)=\sup _{x \in[0, m]^{d}} \int_{[0, m]^{d}} \bar{g}^{2}(y) \sum_{\mathbf{k} \in \mathbb{Z}^{d}} h(x-y-m \mathbf{k}) \mathrm{d} y \leqslant c \int_{[0, m]^{d}} \bar{g}^{2}(y) \mathrm{d} y \leqslant c
$$

using (5.4) and (5.6).

We also have

$$
\mathcal{E}_{\psi, T_{m}^{d}}(\bar{g}, \bar{g})=\int_{[0, m]} \int_{\mathbb{R}^{d}} \frac{|\bar{g}(x+y)-\bar{g}(x)|^{2}}{J_{d+\beta}(y)} \mathrm{d} y \mathrm{~d} x
$$

where the last equality follows as in the proof of (5.3).

Note that by (5.6) we have

$$
\int_{[0, m]^{d}} \bar{g}^{2} * h(x) \mathrm{d} x=\int_{\mathbb{R}^{d}} h(x) \mathrm{d} x<\infty .
$$

Throughout, $c$ will denote a constant which may depend on $h$. Write

$$
E=\bigcup_{i=1}^{d}\left(\left\{0 \leqslant x_{i} \leqslant 2 \sqrt{m}\right\} \cup\left\{m-2 \sqrt{m} \leqslant x_{i} \leqslant m\right\}\right) .
$$

By Lemma 3.4 in Donsker-Varadhan [7], there is an $a \in \mathbb{R}^{d}$ such that

$$
\int_{E} \bar{g}^{2} * h(x+a) \mathrm{d} x \leqslant \frac{c}{\sqrt{m}} .
$$

We may assume $a=0$, i.e.,

$$
\int_{E} \bar{g}^{2} * h(x) \mathrm{d} x \leqslant \frac{c}{\sqrt{m}}
$$


for otherwise we may replace $g(\cdot)$ by $g(a+\cdot)$. Define the function $\phi$ on $\mathbb{R}^{1}$ by

$$
\phi(x)= \begin{cases}x m^{-1 / 2}, & 0 \leqslant x \leqslant m^{1 / 2} \\ 1, & m^{1 / 2} \leqslant x \leqslant m-m^{1 / 2} \\ m^{1 / 2}-x m^{-1 / 2}, & m-m^{1 / 2} \leqslant x \leqslant m, \\ 0, & \text { otherwise }\end{cases}
$$

and write

$$
\begin{aligned}
& \varphi(x)=\phi\left(x_{1}\right) \cdots \phi\left(x_{d}\right), \quad x=\left(x_{1}, \ldots, x_{d}\right) \in \mathbb{R}^{d} \\
& f(x)=\bar{g}(x) \varphi(x) / \sqrt{\int_{\mathbb{R}^{d}} \bar{g}^{2}(y) \varphi^{2}(y) \mathrm{d} y}=\bar{g}(x) \varphi(x) / \sqrt{A} \quad \text { (say). }
\end{aligned}
$$

Then $|\varphi| \leqslant 1,|\nabla \varphi| \leqslant \sqrt{d / m}$ and $f \in \mathcal{F}_{\beta}$.

Note that

$$
\begin{aligned}
|\bar{g}(y) \varphi(y)-\bar{g}(x) \varphi(x)|^{2}= & |(\bar{g}(y)-\bar{g}(x)) \varphi(y)+\bar{g}(x)(\varphi(y)-\varphi(x))|^{2} \\
= & |\bar{g}(y)-\bar{g}(x)|^{2} \varphi^{2}(y)+\bar{g}^{2}(x)|\varphi(y)-\varphi(x)|^{2} \\
& +2|\bar{g}(y)-\bar{g}(x)| \varphi(y) \bar{g}(x)|\varphi(y)-\varphi(x)| .
\end{aligned}
$$

Using

$$
\begin{aligned}
\bar{g}^{2}(x)|\varphi(y)-\varphi(x)|^{2} & \leqslant \bar{g}^{2}(x)|\varphi(y)-\varphi(x)|^{2}\left(1_{[0, m]}(x)+1_{[0, m]^{d}}(y)\right) \\
& \leqslant 2 d m^{-1 / 2} \bar{g}^{2}(x)\left(1_{[0, m]^{d}}(x)+1_{[0, m]^{d}}(y)\right)\left(|y-x| \wedge|y-x|^{2}\right)
\end{aligned}
$$

we find, exactly as in (4.13)-(4.18) of [5] that

$$
\begin{aligned}
A \mathcal{E}_{\psi}(f, f) & =\iint_{\mathbb{R}^{d}} \frac{|\bar{g}(y) \varphi(y)-\bar{g}(x) \varphi(x)|^{2}}{J_{d+\beta}(y-x)} \mathrm{d} y \mathrm{~d} x \leqslant \overline{\mathcal{E}}_{\psi}(\bar{g}, \bar{g})+c m^{-1 / 4} \overline{\mathcal{E}}_{\psi}^{1 / 2}(\bar{g}, \bar{g})+c m^{-1 / 2} \\
& \leqslant\left(1+c m^{-1 / 8}\right) \overline{\mathcal{E}}_{\psi}(\bar{g}, \bar{g})+c m^{-1 / 4} .
\end{aligned}
$$

On the other hand, recalling (5.7)

$$
\begin{aligned}
\left(\int_{T_{m}^{d}}\left(\bar{g}^{2} *_{m} h_{*}(x)\right)^{p} \mathrm{~d} x\right)^{1 / p} & =\left(\int_{[0, m]^{d}}\left|\bar{g}^{2} * h(x)\right|^{p} \mathrm{~d} x\right)^{1 / p} \\
& \leqslant\left(\int_{[0, m]^{d} \backslash E}\left|\bar{g}^{2} * h(x)\right|^{p} \mathrm{~d} x\right)^{1 / p}+\left(\int_{E}\left|\bar{g}^{2} * h(x)\right|^{p} \mathrm{~d} x\right)^{1 / p} .
\end{aligned}
$$

By (5.11) and (5.8)

$$
\left(\int_{E}\left|\bar{g}^{2} * h(x)\right|^{p} \mathrm{~d} x\right)^{1 / p} \leqslant\left(\frac{c}{\sqrt{m}}\right)^{1 / p}\left(\sup _{x \in E} \bar{g}^{2} * h(x)\right)^{\frac{p-1}{p}} \leqslant\left(\frac{c}{\sqrt{m}}\right)^{1 / p} .
$$

Now

$$
\left(\int_{[0, m]}\left|\bar{g}^{2} * h(x)\right|^{p} \mathrm{~d} x\right)^{1 / p}
$$




$$
\leqslant A\left(\int_{\mathbb{R}^{d}}\left|f^{2} * h(x)\right|^{p} \mathrm{~d} x\right)^{1 / p}+\left(\int_{[0, m]^{d} \backslash E}\left|\left(A f^{2}-\bar{g}^{2}\right) * h(x)\right|^{p} \mathrm{~d} x\right)^{1 / p} .
$$

Using the fact that $\left|A f^{2}-\bar{g}^{2}\right| \leqslant \bar{g}^{2}$ and (5.10)

$$
\begin{aligned}
\left(\int_{[0, m]^{d} \backslash E}\left|\left(A f^{2}-\bar{g}^{2}\right) * h(x)\right|^{p} \mathrm{~d} x\right)^{1 / p} & \leqslant\left(\int_{[0, m]^{d}} \bar{g}^{2} * h(x) \mathrm{d} x\right)^{1 / p}\left(\sup _{x \in[0, m]^{d} \backslash E}\left|\left(A f^{2}-\bar{g}^{2}\right) * h(x)\right|\right)^{\frac{p-1}{p}} \\
& \leqslant c\left(\sup _{x \in[0, m]^{d} \backslash E}\left|\left(A f^{2}-\bar{g}^{2}\right) * h(x)\right|\right)^{\frac{p-1}{p}}
\end{aligned}
$$

Also

$$
\sup _{x \in[0, m]^{d} \backslash E}\left|\left(A f^{2}-\bar{g}^{2}\right) * h(x)\right|=\sup _{x \in[0, m]^{d} \backslash E}\left|\int_{\mathbb{R}^{d}}\left(A f^{2}-\bar{g}^{2}\right)(y) h(x-y) \mathrm{d} y\right| .
$$

Since $\left(A f^{2}-\bar{g}^{2}\right)(y)=0$ for all $y \in\left[m^{1 / 2}, m-m^{1 / 2}\right]^{d}$, if also $x \in[0, m]^{d} \backslash E$ we see that the only non-zero contribution to our last integral is for $|x-y| \geqslant m^{1 / 2}$. Hence, using (5.4)

$$
\begin{aligned}
& \sup _{x \in[0, m]^{d} \backslash E}\left|\int_{\mathbb{R}^{d}}\left(A f^{2}-\bar{g}^{2}\right)(y) h(x-y) \mathrm{d} y\right| \\
& \leqslant c \sup _{x \in[0, m]^{d}} \int_{\left\{|x-y| \geqslant m^{1 / 2}\right\}} \bar{g}^{2}(y) \frac{1}{1+|x-y|^{d+\zeta}} \mathrm{d} y \\
& \leqslant c m^{-\zeta / 2} \sup _{x \in[0, m]^{d}} \int_{\left\{|x-y| \geqslant m^{1 / 2}\right\}} \bar{g}^{2}(y) \frac{1}{1+|x-y|^{d+\zeta / 2}} \mathrm{~d} y \\
& \leqslant c m^{-\zeta / 2}
\end{aligned}
$$

where the last inequality follows as in (5.8).

Combining (5.14)-(5.20) and noticing that $A \leqslant 1$ we obtain

$$
\begin{aligned}
& \theta\left(\int_{T_{m}^{d}}\left(\bar{g}^{2} *_{m} h_{*}(x)\right)^{p} \mathrm{~d} x\right)^{1 / p}-\mathcal{E}_{\psi, T_{m}^{d}}(\bar{g}, \bar{g}) \\
& \leqslant A\left(1+\frac{c}{m^{1 / 8}}\right)^{-1}\left\{\theta\left(1+\frac{c}{m^{1 / 8}}\right)\left(\int_{\mathbb{R}^{d}}\left|f^{2} * h(x)\right|^{p} \mathrm{~d} x\right)^{1 / p}-\mathcal{E}_{\psi}(f, f)\right\} \\
& \quad+\theta\left(\frac{c}{\sqrt{m}}\right)^{1 / p}+\theta c m^{-\zeta / 2}+\frac{c}{m^{1 / 4}} \\
& \leqslant M_{\psi, h}\left(\theta\left(1+\frac{c}{m^{1 / 8}}\right)\right)+\theta\left(\frac{c}{\sqrt{m}}\right)^{1 / p}+\theta c m^{-\zeta / 2}+\frac{c}{m^{1 / 4}}
\end{aligned}
$$

where $M_{\psi, h}(\theta)$ denotes the right-hand side of (5.5). Taking the supremum on the left-hand side over $\bar{g} \in \mathcal{F}_{\psi, T_{m}^{d}}$ and then letting $m \rightarrow \infty$ on both sides we have (5.5). 


\section{Exponential approximation}

$$
\begin{aligned}
& \text { Given } \bar{y}=\left(y_{1}, \ldots, y_{p}\right) \in \mathbb{R}^{p d} \text { let } \\
& \alpha_{p, t, \epsilon}(\bar{y})=\int_{0}^{t} \cdots \int_{0}^{t}\left[\int_{\mathbb{R}^{d}}^{p} \prod_{l=1}^{p} h_{\epsilon}\left(X_{l}\left(s_{l}\right)+y_{l}-x\right) \mathrm{d} x\right] \mathrm{d} s_{1} \cdots \mathrm{d} s_{p}
\end{aligned}
$$

where, as before, $h_{\epsilon}(x)=\epsilon^{-d} h(x / \epsilon), h$ is a positive symmetric function in the Schwartz space $\mathcal{S}\left(\mathbb{R}^{d}\right)$ with $\int h \mathrm{~d} x=1$, and $X_{1}(t), \ldots, X_{p}(t)$ are independent $d$-dimensional symmetric stable processes of index $\beta$. We have $\alpha_{p, t, \epsilon}=\alpha_{p, t, \epsilon}(0)$.

Since $h_{\epsilon}(x)=(2 \pi)^{-d} \int_{R^{d}} \mathrm{e}^{\mathrm{i} \lambda \cdot x} \hat{h}(\epsilon \lambda) \mathrm{d} \lambda$ where $\hat{h}$ is the Fourier transform of $h$, we have

$$
\begin{aligned}
\alpha_{p, t, \epsilon}(\bar{y}) & =(2 \pi)^{-d p} \int_{[0, t]^{p}}\left[\int_{\mathbb{R}^{d}}\left(\int_{\mathbb{R}^{d p}} \mathrm{e}^{\mathrm{i} \sum_{l=1}^{p} \lambda_{l} \cdot\left(X_{l}\left(s_{l}\right)+y_{l}-x\right)} \prod_{l=1}^{p} \hat{h}\left(\epsilon \lambda_{l}\right) \mathrm{d} \lambda_{l}\right) \mathrm{d} x\right] \prod_{l=1}^{p} \mathrm{~d} s_{l} \\
& =(2 \pi)^{-d p} \int_{[0, t]^{p}}\left[\int_{\mathbb{R}^{d}} \mathrm{e}^{-\mathrm{i} \sum_{l=1}^{p} \lambda_{l} \cdot x}\left(\int_{\mathbb{R}^{d p}} \mathrm{e}^{i \sum_{l=1}^{p} \lambda_{l} \cdot\left(X_{l}\left(s_{l}\right)+y_{l}\right)} \prod_{l=1}^{p} \hat{h}\left(\epsilon \lambda_{l}\right) \mathrm{d} \lambda_{l}\right) \mathrm{d} x\right] \prod_{l=1}^{p} \mathrm{~d} s_{l} .
\end{aligned}
$$

Using the Fourier inversion formula in the form

$$
(2 \pi)^{-d} \int_{\mathbb{R}^{d}} \mathrm{e}^{-\mathrm{i}\left(\sum_{l=1}^{p-1} \lambda_{l}\right) x} \int_{\mathbb{R}^{d}} \mathrm{e}^{-\mathrm{i} \lambda_{p} x} F\left(\lambda_{p}\right) \mathrm{d} \lambda_{p} \mathrm{~d} x=F\left(-\sum_{l=1}^{p-1} \lambda_{l}\right)
$$

we have that

$$
\alpha_{p, t, \epsilon}(\bar{y})=(2 \pi)^{-d(p-1)} \int_{[0, t]^{p}}\left(\int_{\mathbb{R}^{d(p-1)}} \mathrm{e}^{\mathrm{i} \sum_{l=1}^{p} \lambda_{l} \cdot\left(X_{l}\left(s_{l}\right)+y_{l}\right)} \prod_{l=1}^{p} \hat{h}\left(\epsilon \lambda_{l}\right) \prod_{l=1}^{p-1} \mathrm{~d} \lambda_{l}\right) \prod_{l=1}^{p} \mathrm{~d} s_{l}
$$

where from now on we have $\lambda_{p}=-\left(\sum_{l=1}^{p-1} \lambda_{l}\right)$.

Theorem 8. Let $p(d-\beta)<d$. Then for some $\rho, \theta>0$

$$
\sup _{\epsilon, \epsilon^{\prime}, t>0} E\left(\exp \left\{\theta \frac{\left|\alpha_{p, t, \epsilon}-\alpha_{p, t, \epsilon^{\prime}}\right|^{\beta /(d(p-1)+\rho)}}{\left|\epsilon-\epsilon^{\prime}\right|^{\rho \beta /(d(p-1)+\rho)} t^{p \beta /(d(p-1)+\rho)-1}}\right\}\right)<\infty
$$

and

$$
\sup _{\epsilon, t>0, \bar{y} \neq 0} E\left(\exp \left\{\theta \frac{\left|\alpha_{p, t, \epsilon}-\alpha_{p, t, \epsilon}(\bar{y})\right|^{\beta /(d(p-1)+\rho)}}{|\bar{y}|^{\rho \beta /(d(p-1)+\rho)} t^{p \beta /(d(p-1)+\rho)-1}}\right\}\right)<\infty .
$$

We will prove this theorem shortly, but first note that it follows from our theorem and Kolmogorov's lemma that for any $\bar{y}$

$$
\alpha_{p, t}(\bar{y})=: \lim _{\epsilon \rightarrow 0} \alpha_{p, t, \epsilon}(\bar{y})
$$

exists a.s and in all $L^{q}$ spaces.

It then follows that for some $\rho, \theta>0$

$$
\sup _{\epsilon, t>0, \bar{y}} E\left(\exp \left\{\theta \frac{\left|\alpha_{p, t}(\bar{y})-\alpha_{p, t, \epsilon}(\bar{y})\right|^{\beta /(d(p-1)+\rho)}}{|\epsilon|^{\rho \beta /(d(p-1)+\rho)} t^{p \beta /(d(p-1)+\rho)-1}}\right\}\right)<\infty
$$


and

$$
\sup _{t>0, \bar{y} \neq 0} E\left(\exp \left\{\theta \frac{\left|\alpha_{p, t}-\alpha_{p, t}(\bar{y})\right|^{\beta /(d(p-1)+\rho)}}{|\bar{y}|^{\rho \beta /(d(p-1)+\rho)} t^{p \beta /(d(p-1)+\rho)-1}}\right\}\right)<\infty .
$$

Let us first show how (6.7) leads to Theorem 6.

Proof of Theorem 6. By (6.7) for some $\theta>0$

$$
\sup _{\epsilon, t>0} E \exp \left\{\theta \frac{\left|\alpha_{p, t, \epsilon}-\alpha_{p, t}\right|^{\zeta}}{\epsilon^{\rho \zeta} t^{p \zeta-1}}\right\}<\infty
$$

where

$$
\zeta=\frac{\beta}{d(p-1)+\rho}>\frac{1}{p} .
$$

By the estimate

$$
\begin{aligned}
E \exp \left\{\lambda\left|\alpha_{p, t, \epsilon}-\alpha_{p, t}\right|^{1 / p}\right\} & \leqslant \mathrm{e}^{\lambda \delta t}+E \exp \left\{\lambda\left|\alpha_{p, t, \epsilon}-\alpha_{p, t}\right|^{1 / p}\right\} 1_{\left\{\left|\alpha_{p, t, \epsilon}-\alpha_{p, t}\right| \geqslant \delta^{p} t^{p}\right\}} \\
& \leqslant \mathrm{e}^{\lambda \delta t}+E \exp \left\{\frac{\lambda}{\delta^{p \zeta-1}} \frac{\left|\alpha_{p, t, \epsilon}-\alpha_{p, t}\right|^{\zeta}}{t^{p \zeta-1}}\right\}
\end{aligned}
$$

we conclude that for any $\lambda>0$,

$$
\limsup _{\epsilon \rightarrow 0} \limsup _{t \rightarrow \infty} \frac{1}{t} \log E \exp \left\{\lambda\left|\alpha_{p, t, \epsilon}-\alpha_{p, t}\right|^{1 / p}\right\}=0
$$

which completes the proof of Theorem 6 .

Proof of Theorem 8. Using (6.2) with the convention that $\lambda_{p}=-\left(\sum_{l=1}^{p-1} \lambda_{l}\right)$ we have

$$
\begin{aligned}
& \alpha_{p, t, \epsilon}(\bar{y})-\alpha_{p, t, \epsilon^{\prime}}(\bar{y}) \\
& =(2 \pi)^{-d(p-1)} \int_{[0, t]^{p}}\left(\int_{\mathbb{R}^{d(p-1)}} \mathrm{e}^{\mathrm{i} \sum_{l=1}^{p} \lambda_{l} \cdot\left(X_{l}\left(s_{l}\right)+y_{l}\right)}\left\{\prod_{l=1}^{p} \hat{h}\left(\epsilon \lambda_{l}\right)-\prod_{l=1}^{p} \hat{h}\left(\epsilon^{\prime} \lambda_{l}\right)\right\} \prod_{l=1}^{p-1} \mathrm{~d} \lambda_{l}\right) \prod_{l=1}^{p} \mathrm{~d} s_{l} .
\end{aligned}
$$

Consequently, using the notation $\widehat{H}(\epsilon \lambda ., j)=\prod_{l=1}^{p} \hat{h}\left(\epsilon \lambda_{l, j}\right)$

$$
\begin{aligned}
E\left(\left\{\alpha_{p, t, \epsilon}(\bar{y})-\alpha_{p, t, \epsilon^{\prime}}(\bar{y})\right\}^{m}\right)= & (2 \pi)^{-d m(p-1)} \int_{[0, t]^{m p}} \int_{\mathbb{R}^{d(p-1) m}} E\left(\prod_{l=1}^{p} \mathrm{e}^{\mathrm{i} \sum_{j=1}^{m} \lambda_{l, j} \cdot\left(X_{l}\left(s_{l, j}\right)+y_{l}\right)}\right) \\
& \times \prod_{j=1}^{m}\left(\widehat{H}\left(\epsilon \lambda_{\cdot, j}\right)-\widehat{H}\left(\epsilon^{\prime} \lambda_{\cdot, j}\right)\right)\left(\prod_{l=1}^{p-1} d \lambda_{l, j}\right)\left(\prod_{l=1}^{p} \mathrm{~d} s_{l, j}\right)
\end{aligned}
$$

where from now on

$$
\lambda_{p, j}=-\left(\sum_{l=1}^{p-1} \lambda_{l, j}\right), \quad \forall j=1, \ldots, m .
$$

Since we can write

$$
[0, t]^{m p}=\bigcup_{\pi_{1}, \ldots, \pi_{p}} D_{m}\left(\pi_{1}, \ldots, \pi_{p}\right)
$$


where the union goes over all $p$-tuples of permutations $\pi_{1}, \ldots, \pi_{p}$ of $\{1 \ldots, m\}$, and $D_{m}\left(\pi_{1}, \ldots, \pi_{p}\right)=$ $\left\{\left\{s_{l, j}\right\} \mid 0 \leqslant s_{l, \pi_{l}(1)} \leqslant \cdots \leqslant s_{l, \pi_{l}(m)} \leqslant t, \forall l\right\}$, we can write $(6.13)$ as

$$
\begin{aligned}
E\left(\left\{\alpha_{p, t, \epsilon}(\bar{y})-\alpha_{p, t, \epsilon^{\prime}}(\bar{y})\right\}^{m}\right)= & (2 \pi)^{-d m(p-1)} \sum_{\pi_{1}, \ldots, \pi_{p}} \int_{D_{m}\left(\pi_{1}, \ldots, \pi_{p}\right)} \int_{\mathbb{R}^{d(p-1) m}} E\left(\prod_{l=1}^{p} \mathrm{e}^{\mathrm{i} \sum_{j=1}^{m} \lambda_{l, j} \cdot\left(X_{l}\left(s_{l, j}\right)+y_{l}\right)}\right) \\
& \times \prod_{j=1}^{m}\left(\widehat{H}(\epsilon \lambda,, j)-\widehat{H}\left(\epsilon^{\prime} \lambda_{,}, j\right)\right)\left(\prod_{l=1}^{p-1} \mathrm{~d} \lambda_{l, j}\right)\left(\prod_{l=1}^{p} \mathrm{~d} s_{l, j}\right) .
\end{aligned}
$$

On $D_{m}\left(\pi_{1}, \ldots, \pi_{p}\right)$ we can write

$$
\sum_{j=1}^{m} \lambda_{l, j} \cdot X_{l}\left(s_{l, j}\right)=\sum_{j=1}^{m} u_{l, \pi_{l}, j}\left(X_{l}\left(s_{l, \pi_{l}(j)}\right)-X_{l}\left(s_{l, \pi_{l}(j-1)}\right)\right)
$$

where

$$
u_{l, \pi_{l}, j}=\sum_{i=j}^{m} \lambda_{l, \pi_{l}(i)} .
$$

Hence on $D_{m}\left(\pi_{1}, \ldots, \pi_{p}\right)$

$$
E\left(\prod_{l=1}^{p} \mathrm{e}^{\mathrm{i} \sum_{j=1}^{m} p_{l, j} \cdot X_{l}\left(s_{l, j}\right)}\right)=\prod_{l=1}^{p} F_{l}
$$

where

$$
F_{l}=\mathrm{e}^{-\sum_{j=1}^{m} \psi\left(u_{l, \pi_{l}, j}\right)\left(s_{l, \pi_{l}(j)}-s_{l, \pi_{l}(j-1)}\right)} .
$$

If we set

$$
G_{r}=\prod_{\substack{l=1 \\ l \neq r}}^{p} F_{l}^{1 /(p-1)}
$$

then

$$
E\left(\prod_{l=1}^{p} \mathrm{e}^{\mathrm{i} \sum_{j=1}^{m} \lambda_{l, j} \cdot X_{l}\left(s_{l, j}\right)}\right)=\prod_{r=1}^{p} G_{r} .
$$

Since $\left|\hat{h}\left(\epsilon \lambda_{l, j}\right)-\hat{h}\left(\epsilon^{\prime} \lambda_{l, j}\right)\right| \leqslant C\left|\epsilon-\epsilon^{\prime}\right|^{\rho}\left|\lambda_{l, j}\right|^{\rho}$ for any $\rho \leqslant 1$, we have the bound

$$
\left|\widehat{H}\left(\epsilon \lambda_{,, j}\right)-\widehat{H}\left(\epsilon^{\prime} \lambda_{,}, j\right)\right| \leqslant C\left|\epsilon-\epsilon^{\prime}\right|^{\rho} \sum_{l=1}^{k}\left|\lambda_{l, j}\right|^{\rho} .
$$

Using Hölder's inequality we have

$$
\begin{aligned}
& \left|\int_{\mathbb{R}^{d(p-1) m}} E\left(\prod_{l=1}^{p} \mathrm{e}^{\mathrm{i} \sum_{j=1}^{m} \lambda_{l, j} \cdot\left(X_{l}\left(s_{l, j}\right)+y_{l}\right)}\right) \prod_{j=1}^{m}\left(\widehat{H}\left(\epsilon \lambda_{\cdot, j}\right)-\widehat{H}\left(\epsilon^{\prime} \lambda_{\cdot, j}\right)\right) \prod_{l=1}^{p-1} \mathrm{~d} \lambda_{l, j}\right| \\
& \leqslant C^{m}\left|\epsilon-\epsilon^{\prime}\right|^{\rho m} \int_{\mathbb{R}^{d(p-1) m}} \prod_{r=1}^{p} G_{r} \prod_{j=1}^{m}\left(\sum_{i=1}^{p}\left|\lambda_{i, j}\right|^{\rho}\right) \prod_{l=1}^{p-1} \mathrm{~d} \lambda_{l, j}
\end{aligned}
$$




$$
\leqslant C^{m}\left|\epsilon-\epsilon^{\prime}\right|^{\rho m} \prod_{r=1}^{p}\left(\sum_{i_{1}, \ldots, i_{m}=1}^{p} \int_{\mathbb{R}^{d(p-1) m}} G_{r}^{p} \prod_{j=1}^{m}\left|\lambda_{i_{j}, j}\right|^{\rho} \prod_{l=1}^{p-1} \mathrm{~d} \lambda_{l, j}\right)^{1 / p} .
$$

Absorbing the inner sum in the constant $C^{m}$, we consider

$$
\max _{i_{1}, \ldots, i_{m}} \int_{\mathbb{R}^{d(k-1) m}} G_{r}^{p} \prod_{j=1}^{m}\left|\lambda_{i_{j}, j}\right|^{\rho} \prod_{l=1}^{p-1} \mathrm{~d} \lambda_{l, j} .
$$

If $i \neq r$, then using (6.18) we can bound each $\left|\lambda_{i, j}\right|^{\rho}$ by a sum of two $\left|u_{l, \pi_{l}, j}\right|^{\rho}$ 's and we can be sure that no factor $\left|u_{l, \pi_{l}, j}\right|^{\rho}$ appears more than twice. If $i=r$ then we first use (6.14) to bound each $\left|\lambda_{r, j}\right|^{\rho}$ by a sum of $\left|\lambda_{i, j}\right|^{\rho}$ 's with $i \neq r$, and then proceed as in the last sentence. Thus we can bound (6.25) by

$$
\begin{aligned}
& \max _{h_{r}} C^{m} \int_{\mathbb{R}^{d(p-1) m}} G_{r}^{p} \prod_{j=1}^{m} \prod_{l \neq r}\left|u_{l, \pi_{l}, j}\right|^{\rho h_{r}(l, j)} \mathrm{d} u_{l, \pi_{l}, j} \\
& \leqslant \max _{h_{r}} C^{m} \prod_{\substack{l=1 \\
l \neq r}}^{p} \prod_{j=1}^{m}\left(s_{l, \pi_{l}(j)}-s_{l, \pi_{l}(j-1)}\right)^{-\left(d+\rho h_{r}(l, j)\right) / \beta}
\end{aligned}
$$

where the max runs over the set of functions $h_{r}(l, j)$ taking values $0,1,2,3,4$ such that $h_{r}(r, j)=0$ and $\sum_{l, j} h_{r}(l, j)=m$. Hence

$$
\begin{aligned}
& \left|\int_{R^{d(p-1) m}} E\left(\prod_{l=1}^{p} \mathrm{e}^{\mathrm{i} \sum_{j=1}^{m} \lambda_{l, j} \cdot\left(X_{l}\left(s_{l, j}\right)+y_{l}\right)}\right) \prod_{j=1}^{m}\left(\widehat{H}\left(\epsilon \lambda_{\cdot, j}\right)-\widehat{H}\left(\epsilon^{\prime} \lambda_{\cdot, j}\right)\right) \prod_{l=1}^{p-1} \mathrm{~d} \lambda_{l, j}\right| \\
& \leqslant C^{m}\left|\epsilon-\epsilon^{\prime}\right|^{\rho m} \prod_{r=1}^{p} \max _{h_{r}}\left(\prod_{\substack{l=1 \\
l \neq r}}^{p} \prod_{j=1}^{m}\left(s_{l, \pi_{l}(j)}-s_{l, \pi_{l}(j-1)}\right)^{-\left(d+\rho h_{r}(l, j)\right) / \beta}\right)^{1 / p} \\
& =C^{m}\left|\epsilon-\epsilon^{\prime}\right|^{\rho m} \max _{h_{1}, \ldots, h_{p}} \prod_{l=1}^{p} \prod_{j=1}^{m}\left(s_{l, \pi_{l}(j)}-s_{l, \pi_{l}(j-1)}\right)^{-\left(d(p-1)+\sum_{r=1}^{p} h_{r}(l, j) \rho\right) / k \beta} .
\end{aligned}
$$

Recalling that $p(d-\beta)<d$ so that $d(p-1) / p \beta<1$, hence for $\rho>0$ sufficiently small $d(p-1) / p \beta+4 \rho / \beta<1$, we see that

$$
\begin{aligned}
& \left|E\left(\left\{\frac{\left|\alpha_{p, t, \epsilon}(\bar{y})-\alpha_{p, t, \epsilon^{\prime}}(\bar{y})\right|}{\left|\epsilon-\epsilon^{\prime}\right|^{\rho}}\right\}^{m}\right)\right| \\
& \quad \leqslant C^{m}(m !)^{p} \max _{h_{1}, \ldots, h_{p}} \prod_{l=1}^{p}\left(\int_{0 \leqslant r_{1}<\cdots<r_{m} \leqslant t} \prod_{j=1}^{m}\left(r_{j}-r_{j-1}\right)^{-\left(d(p-1)+\sum_{r=1}^{p} h_{r}(l, j) \rho\right) / p \beta} \mathrm{d} r_{j}\right) \\
& \quad \leqslant C^{m} \max _{h_{1}, \ldots, h_{p}} \prod_{l=1}^{p}\left(\frac{t^{m(1-d(p-1) / p \beta)-\sum_{r=1}^{p} \sum_{j=1}^{m} h_{r}(l, j) \rho / p \beta} m !}{\Gamma\left(m(1-d(p-1) / p \beta)-\sum_{r=1}^{p} \sum_{j=1}^{m} h_{r}(l, j) \rho / p \beta\right)}\right) \\
& \leqslant C^{m} t^{m(p-(d(p-1)+\rho) / \beta)}(m !)^{(d(p-1)+\rho) / \beta} .
\end{aligned}
$$

Writing $\eta=(d(p-1)+\rho) / \beta>1$ we then have

$$
\left|E\left(\left\{\frac{\left|\alpha_{p, t, \epsilon}(\bar{y})-\alpha_{p, t, \epsilon^{\prime}}(\bar{y})\right|}{\left|\epsilon-\epsilon^{\prime}\right|^{\rho} t^{p-\eta}}\right\}^{m}\right)\right| \leqslant C^{m}(m !)^{\eta} .
$$


By Holder's inequality

$$
\left|E\left(\left\{\frac{\left|\alpha_{p, t, \epsilon}(\bar{y})-\alpha_{p, t, \epsilon^{\prime}}(\bar{y})\right|}{\left|\epsilon-\epsilon^{\prime}\right|^{\rho} t^{p-\eta}}\right\}^{m / \eta}\right)\right| \leqslant C^{m}(m !) .
$$

(6.4) follows from this.

To prove (6.5), we note that just as in (6.16) we have

$$
\begin{aligned}
E\left(\left\{\alpha_{p, t, \epsilon}-\alpha_{p, t, \epsilon}(\bar{y})\right\}^{m}\right)= & (2 \pi)^{-d m(p-1)} \sum_{\pi_{1}, \ldots, \pi_{p}} \iint_{D_{m}} E\left(\prod_{l=1}^{p} \mathrm{e}^{\left.\mathrm{i} \sum_{j=1}^{m}, \ldots, \pi_{k}\right)} \lambda_{\mathbb{R}^{d(j, j} \cdot X_{l}\left(s_{l, j}\right)}\right) \\
& \times \prod_{j=1}^{m}\left(1-\mathrm{e}^{\mathrm{i}\left(\sum_{l=1}^{p} \lambda_{l, j} \cdot y_{l}\right)}\right) \widehat{H}\left(\epsilon \lambda_{,, j}\right)\left(\prod_{l=1}^{p-1} \mathrm{~d} \lambda_{l, j}\right)\left(\prod_{l=1}^{p} \mathrm{~d} s_{l, j}\right) .
\end{aligned}
$$

We then proceed as before, but instead of (6.23) we now use the fact that $\|\widehat{H}(\cdot)\|_{\infty} \leqslant C$ and for any $\rho \leqslant 1$, we have the bound

$$
\left|1-\mathrm{e}^{\mathrm{i}\left(\sum_{l=1}^{p} \lambda_{l, j} \cdot y_{l}\right)}\right| \leqslant C|\bar{y}|^{\rho} \sum_{l=1}^{p}\left|\lambda_{l, j}\right|^{\rho} .
$$

The rest of the proof of (6.5) is completed as before. This completes the proof of Theorem 8.

Theorem 9. Let $p(d-\beta)<d$. Then a.s. $\alpha_{p, t, \epsilon}$ converges as $\epsilon \rightarrow 0$ locally uniformly in $t$. Hence, a.s. $\alpha_{p, t}=$ $\lim _{\epsilon \rightarrow 0} \alpha_{p, t, \epsilon}$ is continuous in $t$.

Proof of Theorem 9. Fix $M<\infty$. We will show that for some $\zeta>0$ and any even integer $n$

$$
E\left(\left\{\alpha_{p, t, \epsilon}-\alpha_{p, t^{\prime}, \epsilon^{\prime}}\right\}^{n}\right) \leqslant C_{n}\left|(t, \epsilon)-\left(t^{\prime}, \epsilon^{\prime}\right)\right|^{\zeta n}
$$

uniformly in $t, t^{\prime} \in[0, M]$ and $\epsilon, \epsilon^{\prime} \in(0,1]$. It will then follow from Kolmogorov's Lemma and the fact that $\alpha_{p, t, \epsilon}$ is continuous in $t$ and $\epsilon>0$ that for some $\zeta^{\prime}>0$ and $c_{\omega}<\infty$ a.s.

$$
\left|\alpha_{p, t, \epsilon}-\alpha_{p, t^{\prime}, \epsilon^{\prime}}\right| \leqslant c_{\omega}\left|(t, \epsilon)-\left(t^{\prime}, \epsilon^{\prime}\right)\right|^{\zeta^{\prime}}, \quad \forall t, t^{\prime} \in[0, M], \epsilon, \epsilon^{\prime} \in(0,1]
$$

which implies our theorem.

It thus remains to prove (6.33). We begin with

$$
E\left(\left\{\alpha_{p, t, \epsilon}-\alpha_{p, t^{\prime}, \epsilon^{\prime}}\right\}^{n}\right) \leqslant 2^{n} E\left(\left\{\alpha_{p, t, \epsilon}-\alpha_{p, t^{\prime}, \epsilon}\right\}^{n}\right)+2^{n} E\left(\left\{\alpha_{p, t^{\prime}, \epsilon}-\alpha_{p, t^{\prime}, \epsilon^{\prime}}\right\}^{n}\right) .
$$

From (6.28) we obtain that uniformly in $t^{\prime} \in[0, M]$ and $\epsilon, \epsilon^{\prime} \in(0,1]$

$$
E\left(\left\{\alpha_{p, t^{\prime}, \epsilon}-\alpha_{p, t^{\prime}, \epsilon^{\prime}}\right\}^{n}\right) \leqslant C_{n}\left|\epsilon-\epsilon^{\prime}\right|^{\rho n} .
$$

We may assume that $t^{\prime}<t$. As in (6.16) we have

$$
\begin{aligned}
E\left(\left\{\alpha_{p, t, \epsilon}-\alpha_{p, t^{\prime}, \epsilon}\right\}^{n}\right)= & (2 \pi)^{-d p(n-1)} \sum_{\pi_{1}, \ldots, \pi_{p}} \int_{D_{n}\left(\pi_{1}, \ldots, \pi_{p}\right) \cap\left([0, t]^{p}-\left[0, t^{\prime}\right]^{p}\right)^{n}} \int_{\mathbb{R}^{d p(n-1)}} E\left(\prod_{l=1}^{p} \mathrm{e}^{\mathrm{i} \sum_{j=1}^{n} \lambda_{l, j} \cdot X_{l}\left(s_{l, j}\right)}\right) \\
& \times \prod_{j=1}^{n} \widehat{H}(\epsilon \lambda,, j) \prod_{l=1}^{p-1} \mathrm{~d} \lambda_{l, j} \prod_{l=1}^{p} \mathrm{~d} s_{l, j} .
\end{aligned}
$$

Following through the estimates used in the proof of the last theorem we find that 


$$
\begin{aligned}
& E\left(\left\{\alpha_{p, t, \epsilon}-\alpha_{p, t^{\prime}, \epsilon}\right\}^{n}\right) \\
& \quad \leqslant \sum_{\pi_{1}, \ldots, \pi_{p}} \int_{D_{n}\left(\pi_{1}, \ldots, \pi_{p}\right) \cap\left([0, t]^{p}-\left[0, t^{\prime}\right]^{p}\right)^{n}} \prod_{l=1}^{p} \prod_{j=1}^{n}\left(s_{l, \pi_{l}(j)}-s_{l, \pi_{l}(j-1)}\right)^{-d(k-1) / p \beta} \mathrm{d} s_{l, j} \\
& \quad \leqslant\left\|1_{\left\{\left([0, t]^{p}-\left[0, t^{\prime}\right]^{p}\right)^{n}\right\}}\right\|_{r} \sum_{\pi_{1}, \ldots, \pi_{p}}\left\{\int_{D_{n}\left(\pi_{1}, \ldots, \pi_{p}\right)} \prod_{l=1}^{p} \prod_{j=1}^{n}\left(s_{l, \pi_{l}(j)}-s_{l, \pi_{l}(j-1)}\right)^{-r^{\prime} d(p-1) / k \beta} \mathrm{d} s_{l, j}\right\}^{1 / r^{\prime}}
\end{aligned}
$$

where the last step used Holder's inequality with $\frac{1}{r}+\frac{1}{r}=1$. Take $q>1$ sufficiently close to 1 so that $q d(p-$ $1) / k \beta<1$ and therefore the last integral in (6.38) is bounded. Then we see from (6.38) that for some $\rho^{\prime}>0$

$$
E\left(\left\{\alpha_{p, t, \epsilon}-\alpha_{p, t^{\prime}, \epsilon}\right\}^{n}\right) \leqslant C_{n}\left|t-t^{\prime}\right|^{\rho^{\prime} n}
$$

uniformly in $t, t^{\prime} \in[0, M]$ and $\epsilon \in(0,1]$. This completes the proof of (6.33) and hence of Theorem 9.

\section{The law of the iterated logarithm}

In this section we will prove Theorem 2 . We start with some preparatory material. Given $\bar{y}=\left(y_{1}, \ldots, y_{p}\right) \in$ $\mathbb{R}^{p d}$, we use the notation $P^{\bar{y}}$ and $E^{\bar{y}}$ to denote probabilities and expectations with respect to the $p$ independent symmetric stable processes $X_{1}(t), \ldots, X_{p}(t)$ in $\mathbb{R}^{d}$ of index $\beta$, where now each $X_{j}(0)=y_{j}$. Note that $P^{\bar{y}}\left\{\alpha_{p, t}^{1 / p} \geqslant\right.$ $h t\}=P\left\{\alpha_{p, t}(\bar{y})^{1 / p} \geqslant h t\right\}$ hence

$$
\sup _{|\bar{y}| \leqslant \epsilon} P\left\{\left|\alpha_{p, t}(\bar{y})-\alpha_{p, t}\right|^{1 / p} \geqslant \delta t\right\}+\inf _{|\bar{y}| \leqslant \epsilon} P^{\bar{y}}\left\{\alpha_{p, t}^{1 / p} \geqslant h t\right\} \geqslant P\left\{\alpha_{p, t}^{1 / p} \geqslant(h+\delta) t\right\} .
$$

Using this we see from (1.12) that

$$
\begin{aligned}
& \max \left\{\limsup _{t \rightarrow \infty} \frac{1}{t} \log \sup _{|\bar{y}| \leqslant \epsilon} P\left\{\left|\alpha_{p, t}(\bar{y})-\alpha_{p, t}\right|^{1 / p} \geqslant \delta t\right\}, \liminf _{t \rightarrow \infty} \frac{1}{t} \log \inf _{|\bar{y}| \leqslant \epsilon} P^{\bar{y}}\left\{\alpha_{p, t}^{1 / p} \geqslant h t\right\}\right\} \\
& \geqslant-(h+\delta)^{p \beta / d(p-1)} A_{\psi, p} .
\end{aligned}
$$

Now, from (6.8), as in the proof of (6.11) we find that for any $\lambda>0$,

$$
\limsup _{\epsilon \rightarrow 0} \limsup _{t \rightarrow \infty} \frac{1}{t} \log \sup _{|\bar{y}| \leqslant \epsilon} E \exp \left\{\lambda\left|\alpha_{p, t}(\bar{y})-\alpha_{p, t}\right|^{1 / p}\right\}=0
$$

so that by Chebyshev,

$$
\limsup _{\epsilon \rightarrow 0} \limsup _{t \rightarrow \infty} \frac{1}{t} \log \sup _{|\bar{y}| \leqslant \epsilon} P\left\{\left|\alpha_{p, t}(\bar{y})-\alpha_{p, t}\right|^{1 / p} \geqslant \delta t\right\}=-\infty .
$$

Using this to first let $\epsilon \rightarrow 0$ in (7.2) and then $\delta \rightarrow 0$ we have

$$
\liminf _{\epsilon \rightarrow 0} \liminf _{t \rightarrow \infty} \frac{1}{t} \log \inf _{|\bar{y}| \leqslant \epsilon} P^{\bar{y}}\left\{\alpha_{p, t} \geqslant h t^{p}\right\} \geqslant-h^{\beta / d(p-1)} A_{\psi, p}
$$

By the scaling property $\{X(t s) ; s \geqslant 0\} \stackrel{d}{=} t^{1 / \beta}\{X(s) ; s \geqslant 0\}$ of the stable process which led to (1.7) we have also that

$$
\mathcal{L}^{\bar{y}}\left(\alpha_{p, s t}\right)=\mathcal{L}^{\bar{y} / t^{1 / \beta}}\left(t^{\frac{\beta p-d(p-1)}{\beta}} \alpha_{p, s}\right)
$$

Hence, 


$$
\begin{aligned}
& \liminf _{\epsilon \rightarrow 0} \liminf _{t \rightarrow \infty} \frac{1}{\log \log t} \log \inf _{|\bar{y}| \leqslant \epsilon(t / \log \log t)^{1 / \beta}} P^{\bar{y}}\left\{\alpha_{p, t} \geqslant h t^{\frac{\beta p-d(p-1)}{\beta}}(\log \log t)^{\frac{d(p-1)}{\beta}}\right\} \\
& =\liminf _{\epsilon \rightarrow 0} \liminf _{t \rightarrow \infty} \frac{1}{\log \log t} \log \inf _{|\bar{y}| \leqslant \epsilon} P^{\bar{y}}\left\{\alpha_{\left.p, \log \log t \geqslant h(\log \log t)^{p}\right\}}\right. \\
& =-h^{\beta / d(p-1)} A_{\psi, p} .
\end{aligned}
$$

Proof of Theorem 2. The upper bound follows from (7.7) and a standard use of Borel-Cantelli lemma. We now prove the lower bound. Let $t_{k}=k^{2 k}$ and let

$$
h<A_{\psi, p}^{-\frac{d(p-1)}{\beta}}
$$

We first prove that

$$
\limsup _{k \rightarrow \infty} t_{k+1}^{-\frac{\beta p-d(p-1)}{\beta}}\left(\log \log t_{k+1}\right)^{\frac{-d(p-1)}{\beta}} \alpha_{p}\left(\left(t_{k}, t_{k+1}\right]^{p}\right) \geqslant h \quad \text { a.s. }
$$

By Markov property and Lévy’s Borel-Cantelli lemma, we need only show that

$$
\sum_{k} P^{\widetilde{X}_{t_{k}}}\left\{\alpha_{p, t_{k+1}-t_{k}} \geqslant h t_{k+1}^{\frac{\beta p-d(p-1)}{\beta}}\left(\log \log t_{k+1}\right)^{\frac{d(p-1)}{\beta}}\right\}=\infty \quad \text { a.s. }
$$

where

$$
\widetilde{X}_{t}=\left(X_{1}(t), \ldots, X_{p}(t)\right)
$$

However, it is easy to see that $t_{k+1}-t_{k}>n_{k} k^{2}$ as $k \rightarrow \infty$ so that by the scaling property of the stable process, for any $\delta>0$

$$
P\left(\left|X_{t_{k}}\right|>\frac{\delta\left(t_{k+1}-t_{k}\right)^{1 / \beta}}{\log \log \left(t_{k+1}-t_{k}\right)}\right) \leqslant P\left(\left|X_{1}\right|>\frac{\delta k^{2 / \beta}}{\log k}\right) \leqslant C / k^{2-2 \epsilon}
$$

for any $\epsilon>0$, since $X_{1}$ has $\beta-\epsilon$ moments. By the Borel-Cantelli lemma, with probability 1 the events

$$
\left\{\left|\widetilde{X}_{t_{k}}\right| \leqslant \frac{\delta\left(t_{k+1}-t_{k}\right)^{1 / \beta}}{\log \log \left(t_{k+1}-t_{k}\right)}\right\}, \quad k=1,2, \ldots,
$$

eventually hold. Hence

$$
\lim _{k \rightarrow \infty}\left(\frac{\log \log t_{k+1}}{t_{k+1}}\right)^{1 / \beta}\left|\widetilde{X}_{t_{k}}\right|=0 \quad \text { a.s. }
$$

On the other hand, by (7.7), if $\epsilon>0$ is small enough,

$$
\liminf _{k \rightarrow \infty} \frac{1}{\log \log t_{k+1}} \log \inf _{|\bar{y}| \leqslant \epsilon\left(t_{k+1} / \log \log t_{k+1}\right)^{1 / \beta}} P^{\bar{y}}\left\{\alpha_{p, t_{k+1}-t_{k}} \geqslant h t_{k+1}^{\frac{\beta p-d(p-1)}{\beta}}\left(\log \log t_{k+1}\right)^{\frac{d(p-1)}{\beta}}\right\}>-1 .
$$

Finally the lower bound follows from the relation

$$
\alpha_{p, t_{k+1}} \geqslant \alpha_{p}\left(\left(t_{k}, t_{k+1}\right]^{p}\right)
$$

and the fact that $h$ can be arbitrarily close to $A_{\psi, p}^{-\frac{d(p-1)}{\beta}}$. This completes the proof of Theorem 2. 


\section{Moderate deviations for random walks}

Set

$$
l(n, x, \epsilon)=\sum_{k=1}^{n} b\left(n v_{n}^{-1}\right)^{-d} h_{\epsilon}\left(\frac{S(k)-x}{b\left(n v_{n}^{-1}\right)}\right)
$$

and define $l_{j}(x, n, \epsilon)$ analogously.

Theorem 10. For each $\theta>0$,

$$
\begin{gathered}
\lim _{n \rightarrow \infty} \frac{1}{v_{n}} \log E \exp \left\{\theta \frac{v_{n}}{n} b\left(n v_{n}^{-1}\right)^{\frac{d(p-1)}{p}}\left(\sum_{x \in \mathbb{Z}^{d}} l^{p}(n, x, \epsilon)\right)^{1 / p}\right\} \\
=\sup _{g \in \mathcal{F}_{\psi}}\left\{\theta\left(\int_{\mathbb{R}^{d}}\left|\left(g^{2} * h_{\epsilon}\right)(x)\right|^{p} \mathrm{~d} x\right)^{1 / p}-\mathcal{E}_{\psi}(g, g)\right\}
\end{gathered}
$$

and

$$
\begin{aligned}
& \lim _{n \rightarrow \infty} \frac{1}{v_{n}} \log E \exp \left\{\theta \frac{v_{n}}{n} b\left(n v_{n}^{-1}\right)^{\frac{d(p-1)}{p}}\left(\sum_{x \in \mathbb{Z}^{d}} \prod_{j=1}^{p} l_{j}(n, x, \epsilon)\right)^{1 / p}\right\} \\
& =\sup _{g \in \mathcal{F}_{\psi}}\left\{\theta\left(\int_{\mathbb{R}^{d}}\left|\left(g^{2} * h_{\epsilon}\right)(x)\right|^{p} \mathrm{~d} x\right)^{1 / p}-p \mathcal{E}_{\psi}(g, g)\right\} .
\end{aligned}
$$

Proof of Theorem 10. Given $t>0$, write $t_{n}=\left[t n / v_{n}\right]$ and $\gamma_{n}=\left[n / t_{n}\right]$. Then $n \leqslant t_{n}\left(\gamma_{n}+1\right)$. By independence and the triangle inequality,

$$
\begin{aligned}
E & \exp \left\{\theta \frac{v_{n}}{n} b\left(n v_{n}^{-1}\right)^{\frac{d(p-1)}{p}}\left(\sum_{x \in \mathbb{Z}^{d}} l^{p}(n, x, \epsilon)\right)^{1 / p}\right\} \\
& \leqslant\left(E \exp \left\{\theta \frac{v_{n}}{n} b\left(n v_{n}^{-1}\right)^{-d / p}\left(\sum_{x \in \mathbb{Z}^{d}}\left\{\sum_{k=1}^{t_{n}} h_{\epsilon}\left(\frac{S(k)-x}{b\left(n v_{n}^{-1}\right)}\right)\right\}^{p}\right)^{1 / p}\right\}\right)^{\gamma_{n}+1} \\
& =\left(E \exp \left\{\theta\left(\int_{\mathbb{R}^{d}}\left\{\frac{v_{n}}{n} \sum_{k=1}^{t_{n}} h_{\epsilon}\left(\frac{S(k)-\left[b\left(n v_{n}^{-1}\right) x\right]}{b\left(n v_{n}^{-1}\right)}\right)\right\}^{p} \mathrm{~d} x\right)^{1 / p}\right\}\right)^{\gamma_{n}+1} .
\end{aligned}
$$

Let

$$
\varphi_{n}(f)=\int_{\mathbb{R}^{d}}\left(\int_{0}^{t} h_{\epsilon}\left(f(s)-\frac{\left[b\left(n v_{n}^{-1}\right) x\right]}{b\left(n v_{n}^{-1}\right)}\right) \mathrm{d} s\right)^{p} \mathrm{~d} x .
$$

Applying by Skorohod's generalization of Donsker's invariance principle for random walks in the domain of attraction of a stable process, [9], to the continuous, uniformly bounded and uniformly convergent functionals $\left\{\varphi_{n}(f)\right\}$ on $C\left([0, t] ; \mathbb{R}^{d}\right)$ we have

$$
\lim _{n \rightarrow \infty} E \exp \left\{\theta\left(\int_{\mathbb{R}^{d}}\left\{\frac{v_{n}}{n} \sum_{k=1}^{t_{n}} h_{\epsilon}\left(\frac{S(k)-\left[b\left(n v_{n}^{-1}\right) x\right]}{b\left(n v_{n}^{-1}\right)}\right)\right\}^{p} \mathrm{~d} x\right)^{1 / p}\right\}
$$




$$
=E \exp \left\{\theta\left(\int_{\mathbb{R}^{d}} L^{p}(t, x, \epsilon) \mathrm{d} x\right)^{1 / p}\right\}
$$

where

$$
L(t, x, \epsilon)=\int_{0}^{t} h_{\epsilon}(X(t)-x) \mathrm{d} s .
$$

Therefore,

$$
\begin{aligned}
& \limsup _{n \rightarrow \infty} \frac{1}{v_{n}} \log E \exp \left\{\theta \frac{v_{n}}{n} b\left(n v_{n}^{-1}\right)^{\frac{d(p-1)}{p}}\left(\sum_{x \in \mathbb{Z}^{d}} l^{p}(n, x, \epsilon)\right)^{1 / p}\right\} \\
& \leqslant \frac{1}{t} \log E \exp \left\{\theta\left(\int_{\mathbb{R}^{d}} L^{p}(t, x, \epsilon) \mathrm{d} x\right)^{1 / p}\right\} .
\end{aligned}
$$

Let $t \rightarrow \infty$. By Theorem 7 we have the upper bound,

$$
\begin{gathered}
\limsup _{n \rightarrow \infty} \frac{1}{v_{n}} \log E \exp \left\{\theta \frac{v_{n}}{n} b\left(n v_{n}^{-1}\right)^{\frac{d(p-1)}{p}}\left(\sum_{x \in \mathbb{Z}^{d}} l^{p}(n, x, \epsilon)\right)^{1 / p}\right\} \\
\quad \leqslant \sup _{g \in \mathcal{F}_{\psi}}\left\{\theta\left(\int_{\mathbb{R}^{d}}\left|\left(g^{2} * h_{\epsilon}\right)(x)\right|^{p} \mathrm{~d} x\right)^{1 / p}-\mathcal{E}_{\psi}(g, g)\right\} .
\end{gathered}
$$

By the inequality

$$
\left(\sum_{x \in \mathbb{Z}^{d}} \prod_{j=1}^{p} l_{j}(n, x, \epsilon)\right)^{1 / p} \leqslant \frac{1}{p} \sum_{j=1}^{p}\left(\sum_{x \in \mathbb{Z}^{d}} l_{j}^{p}(n, x, \epsilon)\right)^{1 / p}
$$

and independence,

$$
\begin{aligned}
& \limsup _{n \rightarrow \infty} \frac{1}{v_{n}} \log E \exp \left\{\theta \frac{v_{n}}{n} b\left(n v_{n}^{-1}\right)^{\frac{d(p-1)}{p}}\left(\sum_{x \in \mathbb{Z}^{d}} \prod_{j=1}^{p} l_{j}(n, x, \epsilon)\right)^{1 / p}\right\} \\
& \leqslant \sup _{g \in \mathcal{F}_{\psi}}\left\{\theta\left(\int_{\mathbb{R}^{d}}\left|\left(g^{2} * h_{\epsilon}\right)(x)\right|^{p} \mathrm{~d} x\right)^{1 / p}-p \mathcal{E}_{\psi}(g, g)\right\} .
\end{aligned}
$$

On the other hand, for any nice function $f$ on $\mathbb{R}^{d}$ with $\|f\|_{q}=1$,

$$
\begin{aligned}
\left(\sum_{x \in \mathbb{Z}^{d}} l^{p}(n, x, \epsilon)\right)^{1 / p} & =b\left(n v_{n}^{-1}\right)^{d / p}\left(\int_{\mathbb{R}^{d}} l^{p}\left(n,\left[b\left(n v_{n}^{-1}\right) x\right], \epsilon\right) \mathrm{d} x\right)^{1 / p} \\
& \geqslant b\left(n v_{n}^{-1}\right)^{d / p} \int_{\mathbb{R}^{d}} f(x) l\left(n,\left[b\left(n v_{n}^{-1}\right) x\right], \epsilon\right) \mathrm{d} x \\
& =b\left(n v_{n}^{-1}\right)^{-\frac{d(p-1)}{p}} \sum_{k=1}^{n} \int_{\mathbb{R}^{d}} f(x) h_{\epsilon}\left(\frac{S(k)-\left[b\left(n v_{n}^{-1}\right) x\right]}{b\left(n v_{n}^{-1}\right)}\right) \mathrm{d} x
\end{aligned}
$$




$$
\begin{aligned}
& =b\left(n v_{n}^{-1}\right)^{-\frac{d(p-1)}{p}}\left\{\mathrm{o}(n)+\sum_{k=1}^{n} \int_{\mathbb{R}^{d}} f(x) h_{\epsilon}\left(\frac{S(k)}{b\left(n v_{n}^{-1}\right)}-x\right) \mathrm{d} x\right\} \\
& =b\left(n v_{n}^{-1}\right)^{-\frac{d(p-1)}{p}}\left\{\mathrm{o}(n)+\sum_{k=1}^{n}\left(f * h_{\epsilon}\right)\left(\frac{S(k)}{b\left(n v_{n}^{-1}\right)}\right)\right\} .
\end{aligned}
$$

By the same argument as in Theorem 4.1 of [4],

$$
\begin{gathered}
\liminf _{n \rightarrow \infty} \frac{1}{v_{n}} \log E \exp \left\{\theta \frac{v_{n}}{n} \sum_{k=1}^{n}\left(f * h_{\epsilon}\right)\left(\frac{S(k)}{b\left(n v_{n}^{-1}\right)}\right)\right\} \\
\geqslant \sup _{g \in \mathcal{F}_{\psi}}\left\{\theta \int_{\mathbb{R}^{d}}\left(f * h_{\epsilon}\right)(x) g^{2}(x) \mathrm{d} x-\mathcal{E}_{\psi}(g, g)\right\} \\
=\sup _{g \in \mathcal{F}_{\psi}}\left\{\theta \int_{\mathbb{R}^{d}} f(x)\left(g^{2} * h_{\epsilon}\right)(x) \mathrm{d} x-\mathcal{E}_{\psi}(g, g)\right\} .
\end{gathered}
$$

This gives

$$
\begin{gathered}
\liminf _{n \rightarrow \infty} \frac{1}{v_{n}} \log E \exp \left\{\theta \frac{v_{n}}{n} b\left(n v_{n}^{-1}\right)^{\frac{d(p-1)}{p}}\left(\sum_{x \in \mathbb{Z}^{d}} l^{p}(n, x, \epsilon)\right)^{1 / p}\right\} \\
\geqslant \sup _{g \in \mathcal{F}_{\beta}}\left\{\theta\left(\int_{\mathbb{R}^{d}}\left|\left(g^{2} * h_{\epsilon}\right)(x)\right|^{p} \mathrm{~d} x\right)^{1 / p}-\mathcal{E}_{\psi}(g, g)\right\} .
\end{gathered}
$$

Take $r>0$ and write $B_{r}=\{x|| x \mid \leqslant r\}$. Define

$$
\hat{l}(n, x, \epsilon)=\frac{1}{n} \sum_{k=1}^{n} h_{\epsilon}\left(\frac{S(k)}{b\left(n v_{n}^{-1}\right)}-x\right), \quad x \in B_{r} .
$$

Taking the function $f$ to be supported on $B_{r}=\{x|| x \mid \leqslant r\}$ in the above argument also gives

$$
\begin{aligned}
& \liminf _{n \rightarrow \infty} \frac{1}{v_{n}} \log E \exp \left\{\theta v_{n}\left(\int_{B_{r}} \hat{l}^{p}(t, x, \epsilon) \mathrm{d} x\right)^{1 / p}\right\} \\
& \geqslant \sup _{g \in \mathcal{F}_{\psi}}\left\{\theta\left(\int_{B_{r}}\left|\left(g^{2} * h_{\epsilon}\right)(x)\right|^{p} \mathrm{~d} x\right)^{1 / p}-\mathcal{E}_{\psi}(g, g)\right\} .
\end{aligned}
$$

Then, as in (4.11), there is a compact set $K$ in $L^{p}\left(B_{r}\right)$ such that

$$
\hat{l}(n, \cdot, \epsilon) \in K \quad \text { a.s. } n \geqslant 1 \text {. }
$$

Then the argument used in Section 4 gives

$$
\begin{aligned}
& \liminf _{n \rightarrow \infty} \frac{1}{v_{n}} \log E \exp \left\{\theta v_{n}\left(\int_{B_{r}} \prod_{j=1}^{p} \hat{l}_{j}(t, x, \epsilon) \mathrm{d} x\right)^{1 / p}\right\} \\
& \geqslant \sup _{g \in \mathcal{F}_{\psi}}\left\{\theta\left(\int_{B_{r}}\left|\left(g^{2} * h_{\epsilon}\right)(x)\right|^{p} \mathrm{~d} x\right)^{1 / p}-p \mathcal{E}_{\psi}(g, g)\right\} .
\end{aligned}
$$


Letting $r \rightarrow \infty$ gives

$$
\begin{aligned}
& \liminf _{n \rightarrow \infty} \frac{1}{v_{n}} \log E \exp \left\{\theta v_{n}\left(\int_{\mathbb{R}^{d}} \prod_{j=1}^{p} \hat{l}_{j}(t, x, \epsilon) \mathrm{d} x\right)^{1 / p}\right\} \\
& \geqslant \sup _{g \in \mathcal{F}_{\psi}}\left\{\theta\left(\int_{\mathbb{R}^{d}}\left|\left(g^{2} * h_{\epsilon}\right)(x)\right|^{p} \mathrm{~d} x\right)^{1 / p}-p \mathcal{E}_{\psi}(g, g)\right\} .
\end{aligned}
$$

Notice that

$$
\begin{aligned}
\int_{\mathbb{R}^{d}} & \prod_{j=1}^{p} \hat{l}_{j}(t, x, \epsilon) \mathrm{d} x=\frac{1}{n^{p}} \int_{\mathbb{R}^{d}} \prod_{j=1}^{p} \sum_{k=1}^{n} h_{\epsilon}\left(\frac{S_{j}(k)}{b\left(n v_{n}^{-1}\right)}-x\right) \mathrm{d} x \\
= & \frac{1}{n^{p}} b\left(n v_{n}^{-1}\right)^{-d} \int_{\mathbb{R}^{d}} \prod_{j=1}^{p} \sum_{k=1}^{n} h_{\epsilon}\left(\frac{S_{j}(k)-x}{b\left(n v_{n}^{-1}\right)}\right) \mathrm{d} x \\
= & \frac{\left(1+\mathrm{o}\left(1_{n}\right)\right)}{n^{p}} b\left(n v_{n}^{-1}\right)^{-d} \sum_{x \in \mathbb{Z}^{d}} \prod_{j=1}^{p} \sum_{k=1}^{n} h_{\epsilon}\left(\frac{S_{j}(k)-x}{b\left(n v_{n}^{-1}\right)}\right) \mathrm{d} x \\
= & \frac{\left(1+\mathrm{o}\left(1_{n}\right)\right)}{n^{p}} b\left(n v_{n}^{-1}\right)^{d(p-1)} \sum_{x \in \mathbb{Z}^{d}} \prod_{j=1}^{p} l_{j}(t, x, \epsilon) \mathrm{d} x .
\end{aligned}
$$

Thus

$$
\begin{aligned}
& \liminf _{n \rightarrow \infty} \frac{1}{v_{n}} \log E \exp \left\{\theta \frac{v_{n}}{n} b\left(n v_{n}^{-1}\right)^{\frac{d(p-1)}{p}}\left(\sum_{x \in \mathbb{Z}^{d}} \prod_{j=1}^{p} l_{j}(n, x, \epsilon)\right)^{1 / p}\right\} \\
& \geqslant \sup _{g \in \mathcal{F}_{\beta}}\left\{\theta\left(\int_{\mathbb{R}^{d}}\left|\left(g^{2} * h_{\epsilon}\right)(x)\right|^{p} \mathrm{~d} x\right)^{1 / p}-p \mathcal{E}_{\psi}(g, g)\right\} .
\end{aligned}
$$

This completes the proof of Theorem 10 .

Proof of Theorem 3. We need only prove the first statement, since the second is the consequence of this and Cramer's theorem. For the upper bound we follow some ideas given in [3, Section 5]. For this we need

$$
n^{-p} b(n)^{d(p-1)} I_{p, n} \stackrel{d}{\rightarrow} \alpha_{p, 1} .
$$

This is proven in [8] when $d=2$, and the case of $d=1$ is similar. Let $t_{n}=\left[t n / v_{n}\right]$ and $\gamma_{n}=\left[n / t_{n}\right]+1$. By [3, Theorem 5.1],

$$
\sum_{m=0}^{\infty} \frac{\theta^{m}}{m !}\left(\frac{v_{n}}{n}\right)^{m} b\left(\frac{n}{v_{n}}\right)^{\frac{d(p-1)}{p} m}\left(E\left(I_{p, n}^{m}\right)\right)^{1 / p} \leqslant\left(\sum_{m=0}^{\infty} \frac{\theta^{m}}{m !}\left(\frac{v_{n}}{n}\right)^{m} b\left(\frac{n}{v_{n}}\right)^{\frac{d(p-1)}{p} m}\left(E\left(I_{p, t_{n}}^{m}\right)\right)^{1 / p}\right)^{\gamma_{n}} .
$$

As in [3, Lemma 5.2], there is a positive sequence $\left\{C_{m}\right\}$ such that

$$
\sup _{n}\left(\frac{v_{n}}{n}\right)^{m} b\left(\frac{n}{v_{n}}\right)^{\frac{d(p-1)}{p} m}\left(E\left(I_{p, t_{n}}^{m}\right)\right)^{1 / p} \leqslant C_{m}, \quad m=0,1, \ldots,
$$


and

$$
\sum_{m=0}^{\infty} \frac{\theta^{m}}{m !} C_{m}<\infty
$$

By the weak law and dominated convergence theorem,

$$
\sum_{m=0}^{\infty} \frac{\theta^{m}}{m !}\left(\frac{v_{n}}{n}\right)^{m} b\left(\frac{n}{v_{n}}\right)^{\frac{d(p-1)}{p} m}\left(E\left(I_{p, t_{n}}^{m}\right)\right)^{1 / p} \longrightarrow \sum_{m=0}^{\infty} \frac{\theta^{m}}{m !}\left(E\left(\alpha_{p, t}^{m}\right)\right)^{1 / p}
$$

Hence,

$$
\limsup _{n \rightarrow \infty} \frac{1}{v_{n}} \log \sum_{m=0}^{\infty} \frac{\theta^{m}}{m !}\left(\frac{v_{n}}{n}\right)^{m} b\left(\frac{n}{v_{n}}\right)^{\frac{d(p-1)}{p} m}\left(E\left(I_{p, n}^{m}\right)\right)^{1 / p} \leqslant \frac{1}{t} \log \sum_{m=0}^{\infty} \frac{\theta^{m}}{m !}\left(E\left(\alpha_{p, t}^{m}\right)\right)^{1 / p} .
$$

Let $t \rightarrow \infty$ on the right-hand side of (8.27). By [3, Lemma 5.3] and Theorem 1,

$$
\limsup _{n \rightarrow \infty} \frac{1}{v_{n}} \log \sum_{m=0}^{\infty} \frac{\theta^{m}}{m !}\left(\frac{v_{n}}{n}\right)^{m} b\left(\frac{n}{v_{n}}\right)^{\frac{d(p-1)}{p} m}\left(E\left(I_{p, n}^{m}\right)\right)^{1 / p} \leqslant \sup _{\lambda>0}\left\{\theta \lambda^{1 / p}-p^{-1} A_{\beta, p . d} \lambda^{\beta / d(p-1)}\right\} .
$$

By [3, Lemma 5.3] again, we have the desired upper bound,

$$
\limsup _{n \rightarrow \infty} \frac{1}{v_{n}} \log P\left\{I_{p, n} \geqslant \lambda n^{p} b\left(n v_{n}^{-1}\right)^{d(p-1)}\right\} \leqslant-A_{\beta, p . d} \lambda^{\beta / d(p-1)} .
$$

Recalling (1.13) and the notation $P^{\bar{x}}$ introduced at the beginning of Section 7 we now prove the lower bound:

$$
\begin{gathered}
\liminf _{n \rightarrow \infty} \frac{1}{v_{n}} \log _{|\bar{x}| \leqslant C b(n)} P^{\bar{x}}\left\{I_{p, n} \geqslant \lambda n^{p} b\left(n v_{n}^{-1}\right)^{d(p-1)}\right\} \\
\geqslant-\frac{d(p-1)}{\beta}\left(\frac{\beta p-d(p-1)}{\beta p M_{\psi}}\right)^{\frac{\beta p-d(p-1)}{d(p-1)}} \lambda^{\beta / d(p-1)} .
\end{gathered}
$$

Since the basic idea is given in the proof of [3, Theorem 6.1], we only sketch the argument. To prove the above lower bound, we need only show that

$$
\liminf _{n \rightarrow \infty} \frac{1}{v_{n}} \log \inf _{|\bar{x}| \leqslant C b(n)} E^{\bar{x}} \exp \left\{\theta \frac{v_{n}}{n} b\left(n v_{n}\right)^{\frac{d(p-1)}{p}} I_{p, n}^{1 / p}\right\} \geqslant p^{-\frac{d(p-1)}{p \beta-d(p-1)}} \theta \frac{p \beta}{p \beta-d(p-1)} M_{\psi} .
$$

As in the argument used in [3, Theorem 6.1], we may assume aperiodicity of the random walks and we can then prove, that for any small $u>0$ and $\epsilon>0$, there is a $\delta>0$ and $K>0$ such that

$$
\inf _{|\bar{x}| \leqslant C b(n)} E^{\bar{x}}\left(I_{p, n+[u n]}^{m}\right) \geqslant \delta v_{n}^{-K} E\left(\sum_{x \in \mathbb{Z}^{d}} \prod_{j=1}^{p} l_{j}(n, x, \epsilon)\right)^{m}
$$

for all non-negative integers $m$. This, together with Theorem 10, gives

$$
\begin{gathered}
\liminf _{n \rightarrow \infty} \frac{1}{v_{n}} \log \inf _{|\bar{x}| \leqslant C b(n)} E^{\bar{x}}\left(\exp \left\{\theta \frac{v_{n}}{n} b\left(n v_{n}^{-1}\right)^{\frac{d(p-1)}{p}} I_{p, n+[u n]}^{1 / p}\right\}\right) \\
\geqslant \sup _{g \in \mathcal{F}_{\beta}}\left\{\theta\left(\int_{\mathbb{R}^{d}}\left|\left(g^{2} * h_{\epsilon}\right)(x)\right|^{p} \mathrm{~d} x\right)^{1 / p}-p \mathcal{E}_{\psi}(g, g)\right\} .
\end{gathered}
$$


As $\epsilon \rightarrow 0$, the right-hand side approaches

$$
\sup _{g \in \mathcal{F}_{\beta}}\left\{\theta\left(\int_{\mathbb{R}^{d}}|g(x)|^{2 p} \mathrm{~d} x\right)^{1 / p}-p \mathcal{E}_{\psi}(g, g)\right\}=p^{-\frac{d(p-1)}{p \beta-d(p-1)}} \theta^{\frac{p \beta}{p \beta-d(p-1)}} M_{\psi} .
$$

Notice that $u>0$ can be arbitrarily small. (8.31) then follows by a suitable change of parameters. This completes the proof of Theorem 3 .

Proof of Theorem 4. As in the continuous case, Theorem 4 will follow once we establish

$$
\begin{gathered}
\lim _{\epsilon \rightarrow 0} \liminf _{n \rightarrow \infty} \frac{1}{v_{n}} \log \inf _{|y| \leqslant \epsilon b\left(n v_{n}^{-1}\right)} P^{y}\left\{I_{p, n} \geqslant \lambda n^{p} b\left(n v_{n}^{-1}\right)^{-d(p-1)}\right\} \\
\geqslant-\frac{d(p-1)}{\beta}\left(\frac{\beta p-d(p-1)}{\beta p M_{\psi, p}}\right)^{\frac{\beta p-d(p-1)}{d(p-1)}} \lambda^{\frac{\beta}{d(p-1)}} .
\end{gathered}
$$

This follows from (8.30).

\section{References}

[1] R. Bass, X. Chen, Self intersection local time: critical exponent, large deviations and law of the iterated logarithm, Ann. Probab. 32 (2004) 3221-3247.

[2] R. Bass, X. Chen, J. Rosen, Large deviations for renormalized self-intersection local times of stable processes, Ann. Probab., in press.

[3] X. Chen, Exponential asymptotics and law of the iterated logarithm for intersection local times of random walks, Ann. Probab. 32 (2004) 3248-3300.

[4] X. Chen, W. Li, Large and moderate deviations for intersection local times, Probab. Theory Related Fields 128 (2004) $213-254$.

[5] X. Chen, W. Li, J. Rosen, Large deviations for local times of stable processes and stable random walks in 1 dimension, Electron. J. Probab., in press.

[6] A. Dembo, O. Zeitouni, Large Deviations Techniques and Applications, second ed., Springer, New York, 1998.

[7] M.D. Donsker, S.R.S. Varadhan, On laws of the iterated logarithm for local times, C. P. A. M. XXX (1977) 707-753.

[8] J. Rosen, Random walks and intersection local time, Ann. Probab. 18 (1990) 959-977.

[9] A.V. Skorohod, Limit theorems for stochastic processes, Theory Probab. Appl. 2 (1957) 138-171.

[10] S.J. Taylor, Multiple points for the sample paths of the symmetric stable processes, Z. Wahrsch. Verw. Gebiete 5 (1966) $247-264$. 